\title{
DNA hypomethylation promotes transposable element expression and activation of immune signaling in renal cell cancer
}

\author{
Aguirre A. de Cubas, ${ }^{1}$ William Dunker, ${ }^{2}$ Andrew Zaninovich, ${ }^{1}$ Rachel A. Hongo, ${ }^{1}$ Anuj Bhatia, ${ }^{1}$ \\ Anshuman Panda, ${ }^{3}$ Kathryn E. Beckermann, ${ }^{1}$ Gyan Bhanot, ${ }^{3,4,5,6}$ Shridar Ganesan, ${ }^{3,7}$ John Karijolich, ${ }^{2}$ \\ and W. Kimryn Rathmell ${ }^{1}$ \\ 'Division of Hematology and Oncology, Department of Medicine, Vanderbilt-Ingram Cancer Center, and 'Department of \\ Pathology, Microbiology, and Immunology, Vanderbilt Center for Immunobiology, Vanderbilt University Medical Center, \\ Nashville, Tennessee, USA. ${ }^{3}$ Rutgers Cancer Institute of New Jersey, New Brunswick, New Jersey, USA. ${ }^{4}$ Department of \\ Physics and Astronomy and ${ }^{5}$ Department of Molecular Biology and Biochemistry, Rutgers University, Piscataway, New \\ Jersey, USA. ${ }^{6}$ Moores Cancer Center at UCSD Health, La Jolla, California, USA. ${ }^{7}$ Department of Medicine, Rutgers Robert \\ Wood Johnson Medical School, Rutgers University, Piscataway, New Jersey, USA.
}

Recently, we reported that expression of endogenous retroviruses (ERVs) is associated with response to immune checkpoint blockade (ICB) in renal cell carcinoma (RCC). We show that decitabine, a DNA hypomethylating agent, activates transposable element (TE) expression (LINE1 and ERVs ERV3-2 and ERV4700) and antiviral signaling to potentially enhance response to ICB in kidney cancer cell lines and primary cells. KO of RIGI and MDA5 dsRNA sensors attenuated activation of antiviral signaling associated with DNA hypomethylation, and RIGI and MDA5 IPs showed increased ERV binding with decitabine treatment. Bioinformatic analyses showed the decitabine-induced signature could be associated with increased immune infiltration and response to ICB. Cytokine secretion induced by decitabine could modestly improve $\mathrm{T}$ cell activation and robustly enhanced T cell migration. In a small retrospective cohort of metastatic clear cell RCC (cCRCC) patients treated with anti-PD1/PDL1 blockade, activation of some antiviral genes was significantly higher in responders. Thus, we identified a potential strategy to induce TE expression through inhibition of DNA methylation in modulating $T$ cell action via regulation of the innate antiviral pathway.

Conflict of interest: The authors have declared that no conflict of interest exists.

Copyright: () 2020, American Society for Clinical Investigation.

Submitted: February 24, 2020

Accepted: April 29, 2020

Published: June 4, 2020.

Reference information: /CI Insight. 2020;5(11):e137569.

https://doi.org/10.1172/jici.

insight.137569.

\section{Introduction}

Clear cell renal cell carcinoma (ccRCC) is a common malignancy. The development of immune checkpoint blockade (ICB) for the clinical management of ccRCC has achieved remarkable success in subsets of patients $(1,2)$. However, a minority of ccRCC patients respond to ICB alone. For patients who do not respond to ICB or who develop resistance, the long-term prognosis for survival remains poor (3). Thus, efforts to develop novel strategies to reverse the immunosuppressive microenvironment of ccRCC and sensitize tumors to ICB remains a top priority.

Historically, ccRCC has been considered immunogenic on the basis of observed responsiveness to immune modulating agents $(4,5)$, although the mechanism rendering this response has remained unknown. $\mathrm{CD}^{+} \mathrm{T}$ cell infiltration in advanced ccRCC is associated with a poor risk subgroup $(6,7)$ but with better response to anti-PD1 therapy $(1,8)$. These associations suggest that modulating tumor immunogenicity to increase $\mathrm{T}$ cell infiltration could represent an efficient strategy to promote a set of conditions that allow the tumor immune cells to respond to ICB. Furthermore, while many immune-responsive cancers are characterized by high mutation rates, ccRCCs have modest mutation burden (9). In these regards, we recently reported high expression of endogenous retroviruses (ERVs), a class of transposable element, in a subset of ccRCC tumors, and this can predict response to ICB $(10,11)$. Subsequent evaluation confirmed the association between ERV expression and response to ICB in specimens from a recent clinical trial (12). Thus, ERV expression could at least in part predict ccRCC immunogenicity. 
ERVs are a type of transposable element (TE). TEs have accumulated in our genome over evolution and are estimated to make up at least half of the human genome $(13,14)$. TEs are normally repressed via epigenetic mechanisms, including DNA methylation and histone modification $(15,16)$. Activated ERV expression in cancer cells induces viral mimicry and activates IFN signaling (17-20). Aberrant expression of other TEs has been implicated in autoimmune diseases $(21,22)$. Thus, epigenetic modulation of TE expression might represent a novel therapeutic strategy to enhance immunogenicity and response to ICB. In the current study, we identified a potential strategy to induce TE expression through inhibition of DNA methylation in modulating $\mathrm{T}$ cell action via regulation of the innate antiviral pathway.

\section{Results}

Decitabine, a DNA methylation inhibitor, induces widespread DNA hypomethylation. To assess whether TE expression can be pharmacologically modulated in ccRCC cell lines, we treated a panel of ccRCC cell lines (7860, A498, and UMRC2) and noncancerous transformed kidney cell lines (HKC and renal proximal tubule epithelial [RPTec]) with decitabine, a DNA methylation inhibitor used in the clinic. Cell lines 786-0, A498, and UMRC2 are commonly used for studying cCRCC in vitro and all carry pathogenic mutations in the von Hippel-Lindau (VHL) gene, a defining feature of the vast majority — if not all — of ccRCCs (23-25). RPTec and HKC cells are VHL WT and are representative of normal renal epithelial cells of the proximal tubule. This panel of cell lines permit us to assess the effect of decitabine and TE activation in both cancerous ccRCC cells and normal counterparts in vitro.

To determine appropriate decitabine dose, cytotoxicity was assessed in ccRCC cells using Cell TiterGlo viability assay. As the objective was to induce DNA hypomethylation while minimizing cytotoxicity, decitabine was applied daily for 3 days, and viability was assayed on day 5 . We elected to proceed with 100 $\mathrm{nM}$ and 300-nM doses of decitabine in subsequent experiments (Figure 1A). DNMT1 protein levels were reduced by both 100-nM and 300-nM doses of decitabine (Figure 1, B and C), which resulted in robust and widespread DNA hypomethylation (786-0 cells; Figure 1D and Supplemental Figure 1; supplemental material available online with this article; https://doi.org/10.1172/jci.insight.137569DS1). Although DNA hypomethylation was largely achieved at both doses of decitabine, the mean methylation at a 300$\mathrm{nM}$ dose is lower than observed for the $100-\mathrm{nM}$ dose and shows a tight distribution of methylation values. At the 100-nM dose, the distribution of DNA methylation values was wider than the 300-nM dose and shows a long tail at higher DNA methylation levels. This regimen allowed for maximal DNA hypomethylation while minimizing cytotoxicity.

DNA hypomethylation can modulate TE expression in ccRCC cells. We treated the 786-0 ccRCC cells and normal HKC cells with DMSO or decitabine and performed RNA sequencing (RNAseq) to assess global gene expression (see below). Although RNAseq-based quantification of TE expression is not optimal using poly-A-selected RNA libraries (26), a total of 1176 TEs were detected by RNAseq in our samples (HKC and 786-0; $n=12$; Supplemental Table 1). Unsupervised analysis of TE expression levels separated samples according to decitabine treatments, as well as cell line (Figure 2A). Unsupervised analysis shows robust TE activation in decitabine-treated 786-0 cells, while TE expression was modestly induced by decitabine in HKC cells. Interestingly, the unsupervised analysis showed that untreated 786-0 ccRCC cells have similar levels of TE expression as untreated and treated HKC kidney cells. This suggests that TE activation is attenuated in normal kidney cells in comparison with ccRCC cells.

Supervised analysis was performed separately for 786-0 and HKC cell lines to identify differentially expressed TEs between decitabine (100 nM and $300 \mathrm{nM})$ and vehicle (DMSO) treatment groups. For RNAseq-based quantification of TE expression, we allowed for inclusion of multimapping reads to account for their repetitive nature and potential presence at multiple locations in the genome. During alignment, multimapping reads were randomly assigned to corresponding regions. As a result, differences in expression can be subtle for TEs, in contrast to canonical genes that are easily mapped (aligned) and usually present once in the genome. For these reasons, the fold change cutoff was lowered for differentially expressed TEs ( $\mid \log _{2}$-fold $\mid>0.75$ ). In total, 41 and 119 TEs were significantly induced by decitabine in HKC and 786-0 cells, respectively (2-tailed ANOVA, FDR < 0.05 and $\mid \log _{2}$-fold $\mid>0.75$; Supplemental Tables 2 and 3 ).

Differentially expressed TEs were classified according to Repbase-defined groups (27) (ERV and long terminal repeat retrotransposon [LTR-retrotransposon], DNA transposon, non-LTR-retrotransposon, and unclassified/ancient elements; Supplemental Tables 2 and 3). In both HKC and 786-0 cells, ERVs and LTR-retrotransposons were the most abundant class of differentially expressed TEs, followed by 
A

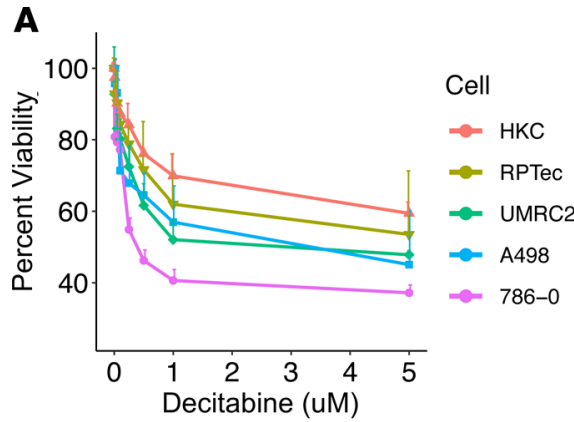

B

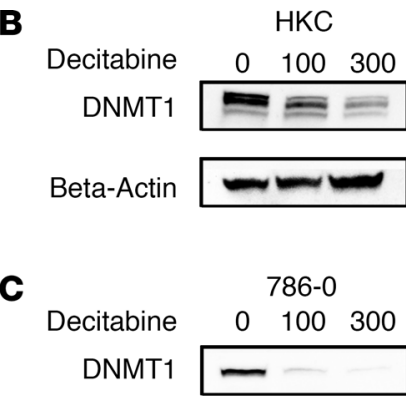

Beta-Actin

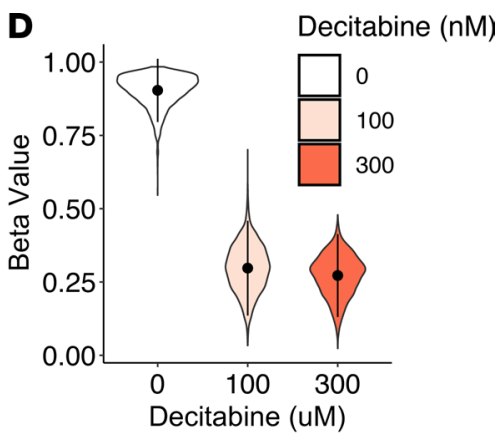

Figure 1. Decitabine induces DNA hypomethylation in ccRCC cell lines. Kidney cell lines were treated with the indicated doses of decitabine for 3 consecutive days and assayed on day 5. (A) Decitabine dose-response curve for viability in panel of kidney cell lines (A498, HKC, RPTec, UMRC2, and 786-0). All data are mean \pm SD $(n=3)$. (B and $\mathbf{C})$ DNMT1 protein levels were assessed in HKC (B) and 786-0 (C) by immunoblot analysis. $\beta$-Actin was included as a loading control. (D) DNA methylation levels were assayed in 786-0 cells treated with decitabine. Violin plot showing distribution of DNA methylation patterns for the 50,000 most variably methylated probes. Black dot and line at violin center indicate mean \pm SD. Data represent the mean of duplicate samples.

DNA transposons (Figure 2, B and C). To validate TE RNAseq data showing upregulation of a number of ERVs and LTR-retrotransposons, we quantified expression of several ERVs (ERV3-2, ERV4700, ERVFC1, and ERVFC2) by quantitative PCR (qPCR) in ccRCC cell lines (786-0, A498, and UMRC2) and normal HKC cells treated with decitabine or DMSO. ERV3-2, ERVFC1, and ERVFC2 were selected for validation, as these were robustly induced by DNA hypomethylation according to our RNAseq data, while ERV4700 and ERV3-2 were selected for validation on the basis that these ERVs were previously identified as markers for response to ICC in CCRCC (10-12). Additionally, ERVFC1 and ERVFC2 have been previously described as regulated by DNA methylation $(17,18)$ and would serve as a control. Although decitabine induced expression of these ERVs in a dose-dependent manner in all cell lines, ERV induction was attenuated in the normal HKC cells in comparison with ccRCC cells (Figure 2D and Supplemental Figure 2). Interestingly, 786-0 cells expressed a high proportion of non-LTR-retrotransposons, which include long interspersed nuclear elements (LINEs) and short interspersed nuclear elements (SINEs), compared with noncancerous HKC cells. Increased expression of LINE-1 protein (ORFp1) was verified at the 300-nM dose (Figure 2E), but not at $100 \mathrm{nM}$ (Supplemental Figure 3) in 7860 cells. Although both 100-nM and 300-nM doses of decitabine induced robust DNA hypomethylation, our results suggest that there is a dosage effect in many of the responses. Decitabine treatment did not induce ORFp1 expression in HKC cells at either dose (Supplemental Figure 4), consistent with RNAseq data. These results show that DNA hypomethylation induces more robust TE expression in 786-0 ccRCC cells than in normal HKCs, which resembles levels in untreated 786-0 cells.

Although the majority of TEs were induced by decitabine, approximately $15 \%-25 \%$ of TEs were downregulated (Figure 2F). This suggests that DNA methylation is not the only means governing TE expression or that compensatory mechanisms can repress TEs following DNA hypomethylation. To assess relationships between DNA hypomethylation and TE expression, we performed an integrative analysis between the 2 data sets. We defined promoter regions as 2000 bases upstream and 400 bases downstream for the 119 TEs differentially expressed by $300 \mathrm{nM}$ decitabine treatment in 786-0 cells (see below), of which data are available for 92. Integration of DNA methylation and TE expression levels show a significant inverse relationship (Figure $2 \mathrm{G}$, Pearson correlation $=-0.62 ; P<2.2 \times 10^{-16}$ ). These results suggest DNA hypomethylation is activating TE expression.

Activated TE expression triggers antiviral signaling. Looking to alterations in expression beyond TEs, HKC cells treated with $100 \mathrm{nM}$ and $300 \mathrm{nM}$ decitabine showed a total of 317 and 1058 differentially expressed genes, respectively (FDR < 0.05; ( $\mid \log _{2}$ fold $\mid>1.5$; Supplemental Tables 4 and 5). In 786-0, a total of 3158 and 4264 genes were differentially expressed by $100 \mathrm{nM}$ and $300 \mathrm{nM}$ decitabine, respectively (FDR < 0.05; ( $\mid \log _{2}$ fold $\mid>1.5$; Supplemental Tables 6 and 7). The difference in number of differentially expressed genes between the 100 and $300 \mathrm{nM}$ dose in HKC ( $n=317$ and $n=1058$, respectively) and 786-0 ( $n=3158$ and $n$ $=4264$, respectively) cell lines was striking (Table 1 ). Similar to TE expression, decitabine treatment generally led to increased gene expression (Supplemental Figures 5 and 6), although some were downregulated. Functional annotation was performed focusing on genes significantly upregulated by decitabine treatment. 
A

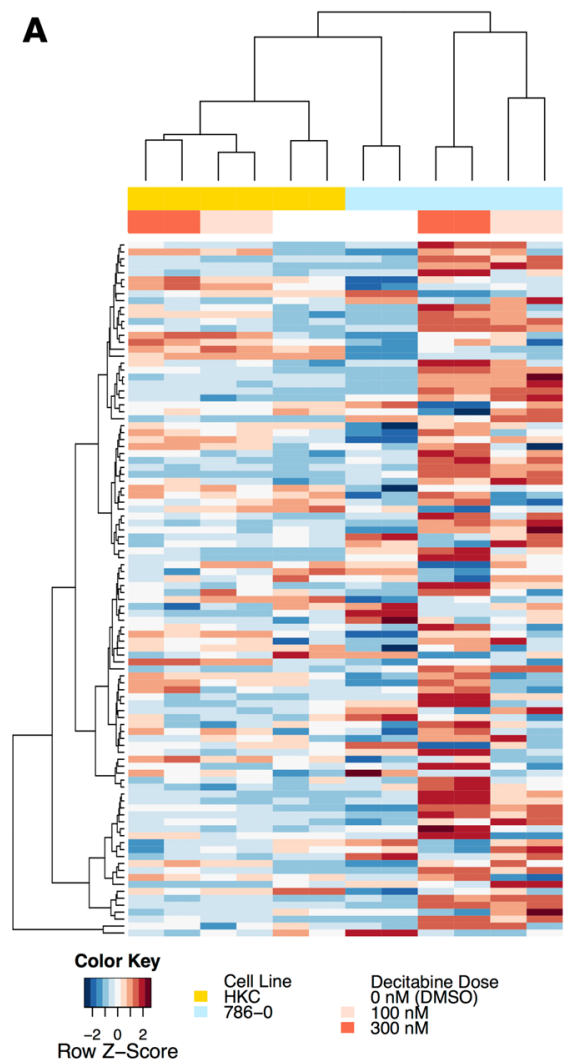

B

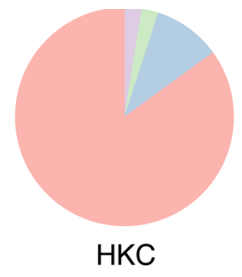

ERV3-2
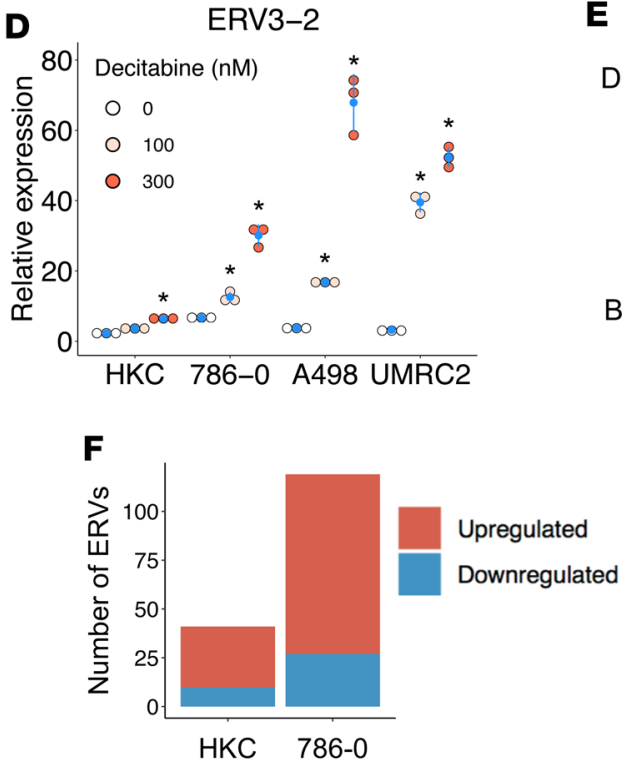

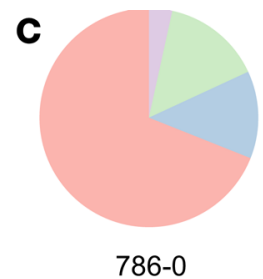

$\mathbf{E}$

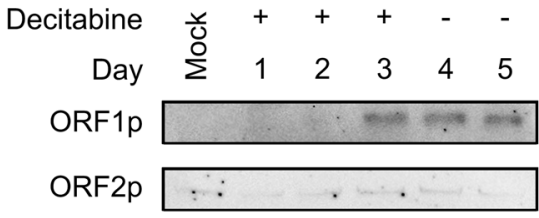

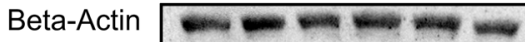

Figure 2. DNA hypomethylation activates TE expression ccRCC cells. (A) Heatmap visualization of unsupervised hierarchal clustering for the 100 most variably expressed TEs. HKC and 786-0 cells were treated with indicated doses of decitabine, and TE expression was assessed by RNAseq (performed in duplicate $[n=2]$ for each condition). (B and C) Pie chart showing distribution of differentially expressed TE classes for HKC (B) and 786-0 (C). (D) Expression of ERV3-2 in a panel of kidney cell lines by qPCR. Blue dot and line indicate mean \pm SD $(n=3)$. Significance assessed by 2-tailed $t$ test, and $P$ values were adjusted via Holm-Bonferroni correction. ${ }^{*} P<0.05$ by Bonferroni correction. (E) Expression of LINE-1 ORFp1 and ORFp2 proteins assessed by immunoblot analysis. $\beta$-Actin included as a loading control. 786-0 cells were treated with $300 \mathrm{nM}$ decitabine for 3 days (days 0,1 , and 2), and protein was harvested at days 1-5. Mock-treated cells were treated with DMSO for 3 days (days 0, 1, and 2), and protein was harvested at day 5. (F) Stacked bar plot showing significantly upregulated (red) and downregulated (blue) transposable elements in HKC and 786-0 cell lines (RNAseq; Wald test, FDR $<0.05$ and |log ${ }_{2}$-fold| $>0.75$ ). (G) Scatterplot shows relationship between DNA methylation and TE expression for differentially expressed TEs ( $n=92$ ) in 786-0 cells (RNAseq). Red triangles and gray dots represent data points for the $300 \mathrm{nM}$ decitabine or DMSO treatment group, respectively. Blue dotted line shows linear model fit to data (Pearson correlation $=-0.62 ; P<2.2 \times 10^{-16}$ ). Data represent the mean of duplicate samples.

Upregulated genes showed significant enrichment in many immune-related pathways (Figure 3A and Supplemental Figure 7). Robust upregulation of canonical components of the antiviral pathway, including pattern recognition receptors involved in sensing nucleic acids (DDX58 [RIGI], IFIH1 [MDA5], DHX58 [LGP2]) and downstream effectors (IRF7, ISG15, OAS3, and MX1), was observed in decitabine-treated cells. Antiviral signaling was activated at both doses of decitabine in 786-0 and HKC cells (Figure 3B and Supplemental Figure 8). As observed for TE expression patterns, antiviral signaling in HKCs was much less in magnitude compared with 786-0 cells, as verified by qPCR and NanoString (pan-cancer immune panel) analyses (Supplemental Figures 9 and 10 and Supplemental Table 8).

Activated TE expression and antiviral signaling is long lasting. To verify that the upregulation of antiviral signaling leads to increased protein levels, we assessed expression of DNMT1 and canonical antiviral proteins (MDA5, RIGI, IRF7, and ISG15) over 5 days during decitabine treatment (Figure 3C and Supplemental Figures 11 and 12). Reduced DNMT1 protein was evident at 24 hours after decitabine treatment (100 and $300 \mathrm{nM}$, respectively, in HKC and 786-0). DNMT1 levels remain low until 24 hours after the last decitabine treatment (day 4), when protein levels begin to recuperate at day 5. Antiviral protein (MDA5, RIGI, IRF7, and ISG15) levels showed an inverse pattern of expression compared with DNMT1. At 24 hours, levels of antiviral proteins are very low. Their expression begins to increase at day 2 and reaches maximum levels at day 4. It is important to note that measurements of TEs, as well as gene expression levels and DNA methylation levels, were carried out at 48 hours after the last decitabine treatment (day 5). At day 5, DNMT1 
Table 1. Summary of differentially expressed genes in decitabine treated kidney cells

\begin{tabular}{|c|c|c|c|c|}
\hline Cell line & Decitabine (nM) & Total genes $($ FDR < 0.05 ) & Upregulated genes & Downregulated genes \\
\hline HKC & 100 & 317 & 297 & 20 \\
\hline $\mathrm{HKC}$ & 300 & 1058 & 977 & 81 \\
\hline $786-0$ & 100 & 3158 & 2227 & 931 \\
\hline 786-0 & 300 & 4264 & 3924 & 340 \\
\hline
\end{tabular}

HKC and 786-0 cells were treated with the indicated doses of decitabine. Indicated numbers of genes were significantly expressed between indicated decitabine-treatment group and respective DMSO control (Wald test, FDR $<0.05$ and $\mid \log _{2}$-fold $\mid>1.5$ ).

protein levels began to recuperate, antiviral proteins remained elevated, and activation of TE expression and antiviral signaling continued to be high, while DNA remained hypomethylated at this time point. This is most evident at the 100-nM dose in both HKC (Supplemental Figure 12A) and 786-0 (Figure 3C) cells. These results suggest that reprograming of DNA methylation induced by decitabine is long lasting.

RIGI and MDA5 bind ERVs induced by DNA hypomethylation to activate antiviral signaling in ccRCC cells. To determine if ERV expression induced by DNA hypomethylation is required for the activation of antiviral signaling, we individually knocked out MAVS, RIGI, and MDA5 using CRISPR/Cas9 in 786-0 cells (Figure 4A). These cells were treated with low-dose decitabine (100 nM), and antiviral signaling was assessed by qPCR. KO of MDA5 and RIGI attenuated activation of ISG15, MX1, and OAS3, while IRF7 expression was not affected (Figure 4, B-E). ERV activation was verified in these cells (Figure 4, F and G). Accordingly, these results were recapitulated by MAVS KO, which signals downstream of RIGI and MDA5. To investigate whether ERVs induced by DNA hypomethylation are bound by RIGI and MDA5, we performed RNA IP (RIP) of Doxyline-inducible FLAG-tagged RIGI and MDA5 protein constructs in 786-0 cells treated with decitabine or DMSO. On day 2, cells were treated with Doxyline to induce expression of the FLAG-tagged RIGI or MDA5 protein construct (Figure 5A). After IP, isolated RNAs were quantified by qPCR for ERVE, ERVFC1, ERVFC2, ERV3-2, and ERV4700 (Gag 1, Pol 2, and Env 1 regions). Enrichment of ERVs bound by RIGI and MDA5 was increased by decitabine treatment and dependent on the ERV species (Figure 5, B-E). These results indicate that ERVs activated by DNA hypomethylation contribute to the induction of antiviral signaling through activation of the MDA5/RIGI/MAVS cascade.

Decitabine-induced expression patterns correspond to activated immunity in tumor specimens. To determine whether decitabine-induced activation of TE expression and antiviral responses could promote immunogenicity in cCRCC, we performed a univariate linear regression to identify significant associations between decitabine-induced genes $(786-0 ; 0-300 \mathrm{nM}$ decitabine; $n=3924)$ and immune cell infiltrate using The Cancer Genome Atlas (TCGA) ccRCC RNAseq data as a training data set $(25,28)$. We used ESTIMATE Immune Score as a surrogate for immune infiltration (29). In total, 567 genes showed significant $(F D R<0.05)$ and positive associations with immune infiltrate. For validation, we tested these genes in an independent gene expression data set (30). A total of 355 genes showed significant association with immune infiltrate, suggesting that decitabine (by proxy of genes induced by treatment) could enhance immunogenicity in ccRCC. Finally, we asked whether specifically antiviral genes, associated with decitabine treatment, were associated with immune infiltrate. Using TCGA ccRCC RNAseq data, we performed an unsupervised hierarchal analysis to cluster samples according to expression levels of 24 antiviral genes (Figure 6A). Samples formed 2 clusters. One cluster, showing increased expression of these 24 antiviral genes, was highly enriched with tumors with high immune infiltrate. The other cluster formed 2 subclusters. One had low levels of expression of these genes and contained tumors with low infiltrate. The other subcluster contained a mixture of high- and low-infiltrate tumors and showed intermediate levels of expression of the 24 antiviral genes. These results suggest that expression of antiviral genes is associated with increased immune infiltrate.

To verify that the association between expression of antiviral genes and immune infiltrate per the ESTIMATE Immune Score was not due to a tautological relationship, we assessed whether this association withheld using percent immune infiltrate as determined by pathological evaluation of histology slides available for TCGA samples. A linear regression between the mean expression of antiviral genes and histology immune infiltrate showed a significant and positive association (Figure 6B; Pearson 
A

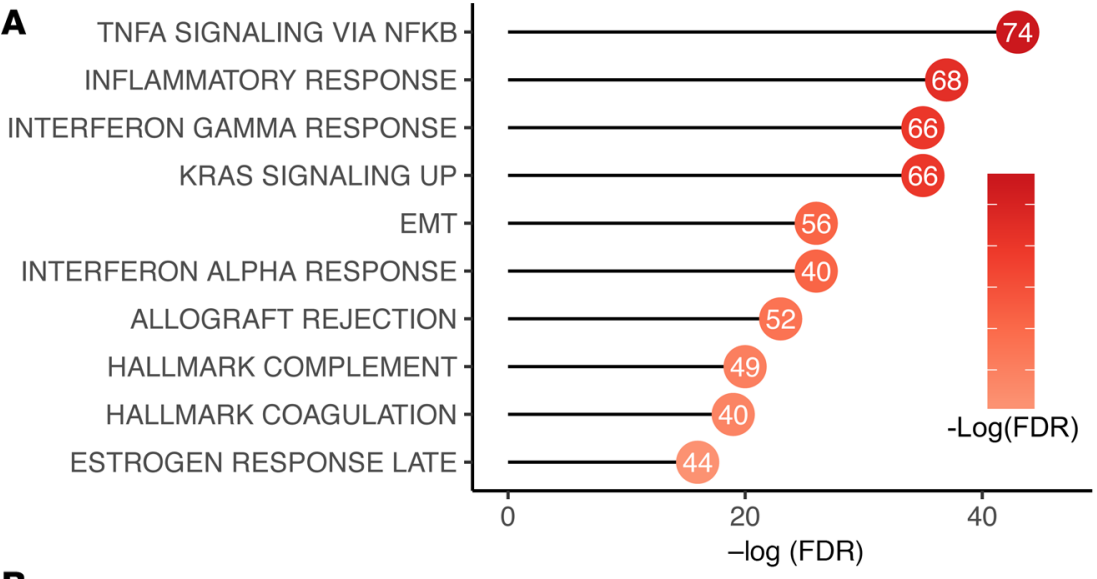

B

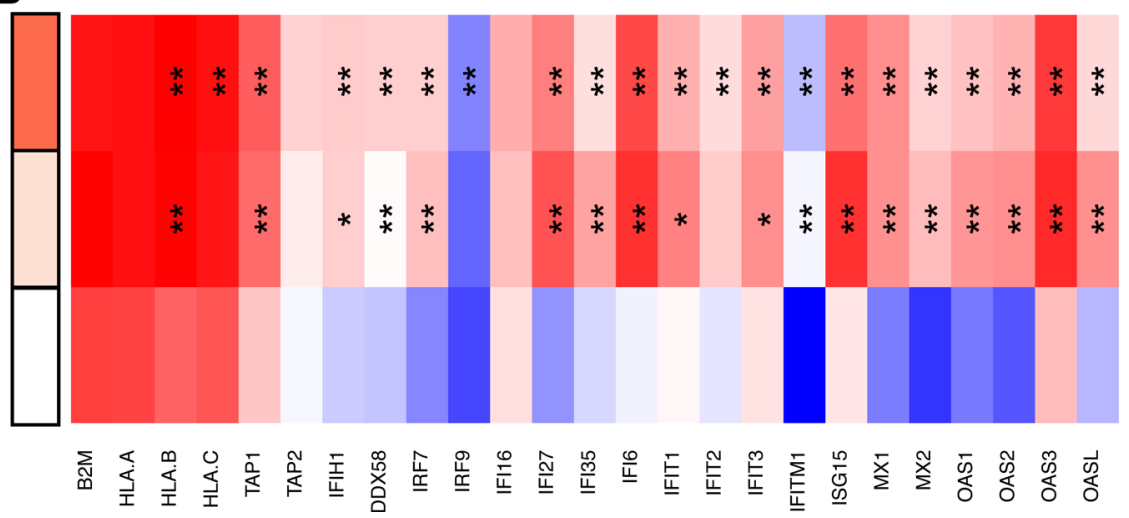

C

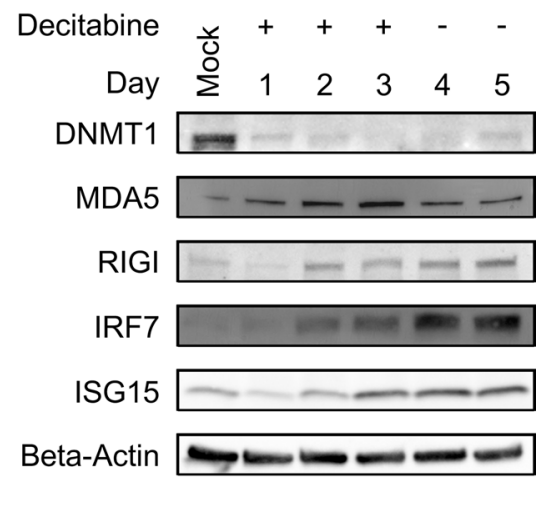

Figure 3. Decitabine treatment activates antiviral signaling in ccRCC cells. 786-0 cells were cultured for 3 days with decitabine, cells were harvested on day 5 and assayed by RNAseq. RNAseq was performed in duplicate samples $(n=2)$ for each experimental condition. (A) Lollipop representation of significantly enriched pathways for genes upregulated by decitabine treatment (300 nM) in 786-0 cells as compared with DMSO vehicle (RNA-Seq; Wald test, FDR $<0.05$ and $\log _{2}$-fold $>1.5$ ). Lollipop height and intensity color scale indicate magnitude -log ${ }_{10}$ (FDR) of the enrichment for each pathway. The number of genes enriched in that pathway is indicated inside each lollipop gene enriched in that pathway. (B) Expression levels of antiviral response genes in 786-0 cells treated with $0 \mathrm{nM}, 100 \mathrm{nM}$, and $300 \mathrm{nM}$ decitabine. Heatmap rows show mean expression of duplicate RNAseq measurements. Significance (100 nM versus $0 \mathrm{nM} ; 300 \mathrm{nM}$ versus $0 \mathrm{nM}$ ) assessed by Wald test and corrected for multiple testing (FDR). ${ }^{*}$ FDR $<0.05$ and ${ }^{*}{ }^{*}$ FDR $<0.001$. (C) Antiviral protein levels were assessed by immunoblot analysis. 786-0 cells were treated with 100 nM decitabine for 3 days (days 0,1 , and 2 ), and protein was harvested at days 1-5. Mock-treated cells were cultured with DMSO for 3 days, and protein was harvested at day 5. Protein expression levels of DNMT1, RIGI, MDA5, IRF7, ISG15, and $\beta$-actin were assessed by immunoblot. $\beta$-Actin was used as a loading control.

correlation $\left.=0.41 ; P=2.28 \times 10^{-6}\right)$. These results suggest that decitabine treatment, by virtue of TE reexpression and activation of antiviral signaling, could promote immunogenicity in ccRCCs.

DNA hypomethylation induces TEs and antiviral signature in ccRCC patient samples. To determine whether decitabine-induced TE expression has translational potential in the clinical treatment of ccRCC, we quantified ERV expression and antiviral signaling in patient-derived primary ccRCC cell lines $(n=5)$ treated with decitabine and DMSO. Consistently, ccRCC primary cells showed upregulation of ERV expression (Figure 7, A-C) and antiviral signaling in response to decitabine (Figure 7D). These results show that decitabine can activate TEs and antiviral signaling in not only ccRCC cell lines, but also in primary ccRCC cells.

Antiviral signaling is associated with response to ICB therapy. To examine whether there is an association between activation of antiviral signaling and response to ICB, we analyzed antiviral signaling in a cohort of ccRCC samples $(n=24)$ from patients who responded ( $n=13$; partial response and progression-free survival of at least 6 months) and that did not respond ( $n=11$; immediate progressive disease) to single-agent anti-PD1 therapy. Clinical and demographic data for patient cohorts are available in Supplemental Table 9. Interestingly, $D D X 58, I F I H 1$, and $D H X 58$ (LGP2) expression was upregulated in responders $(P=0.006, P=$ 0.011 , and $P=0.027$, respectively) (Figure 8, A-C). Upregulation of $I R F 7$ and $I S G 15$ in responders did not reach significance $(P=0.24, P=0.32$; Figure $8, \mathrm{D}$ and $\mathrm{E})$. These results suggest that activation of antiviral signaling (DDX58,DHX58, and IFIH1) could be a potential marker for response to ICB. 


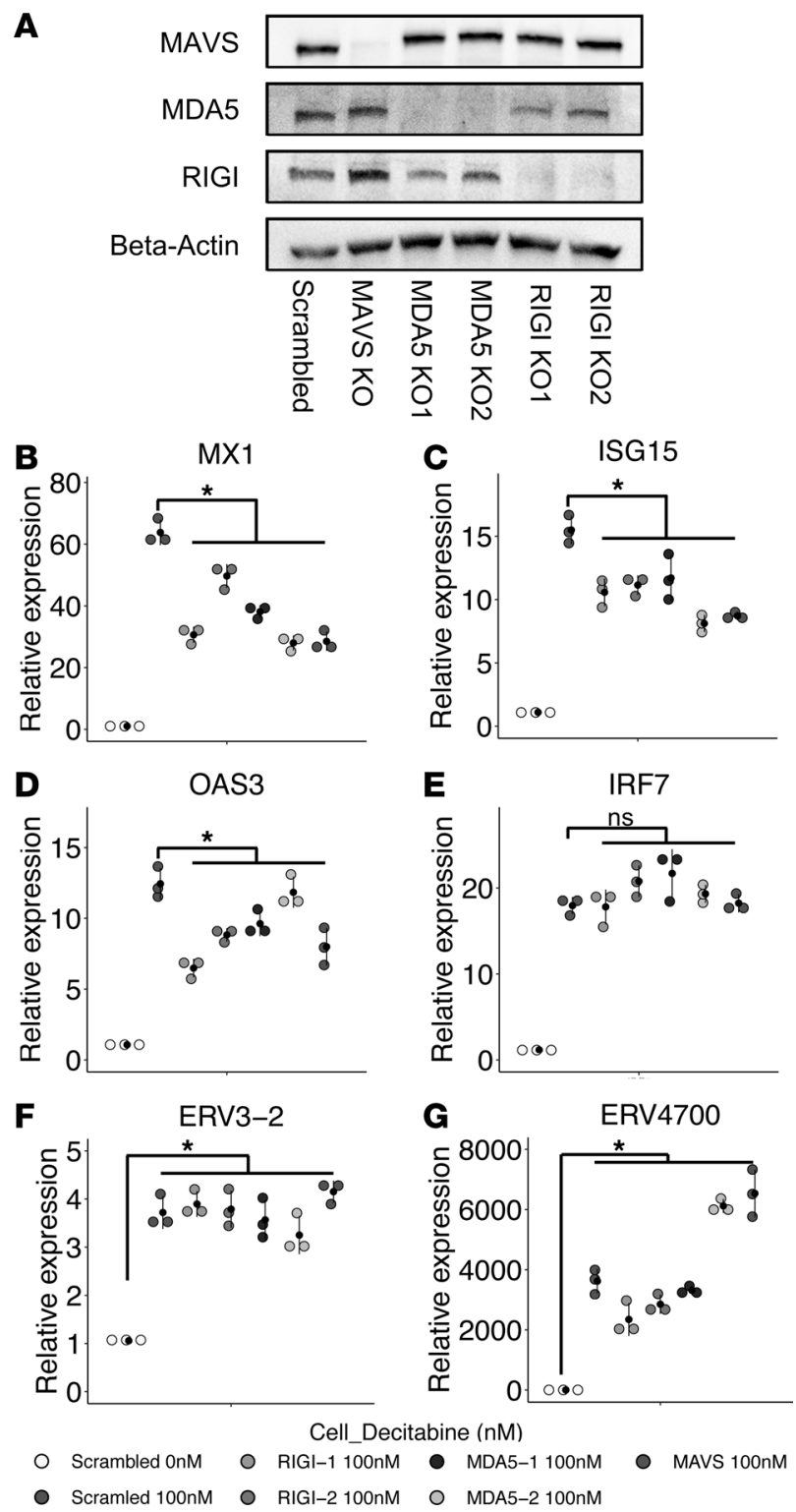

Figure 4. KO of MDA5, RIGI, or MAVS attenuates antiviral signaling in ccRCC cells. (A) Verification of MAVS-, MDA5-, and RIGI-KO cells in indicated 786-0 cell lines by immunoblot analysis. $\beta$-Actin was used as a loading control. (B-E) Antiviral signaling is attenuated by MDA5-, RIGI-, or MAVS-KO 786-0 cells, but not in scrambled control cells. Antiviral signaling was assessed by qPCR of IRF7, ISG15, MX1, and OAS3 genes. Black dot and line indicate mean \pm SD $(n=3)$. Significance (MAVS/RIGI/MDA5-KO [100 nM] versus Scramble [100 nM]) assessed by 2-tailed $t$ test, and $P$ values were adjusted via Holm-Bonferroni correction. ${ }^{*} P<0.05$. (F and $\left.\mathbf{G}\right)$ ERV expression is activated by decitabine treatment of in MAVS-, MDA5-, and RIGI-KO in 786-0 cell lines and scrambled controls. ERV3-2 and ERV4700 expression measured by qPCR.

$D N A$ hypomethylation and TE expression induces cytokine secretion and antiviral signaling in naive $T$ cells. To assess whether upregulation of inflammatory cytokine genes, per our RNAseq and NanoString data, corresponded to increased secretion, we quantified levels of cytokines secreted in culture media by $786-0$ cells treated with DMSO and decitabine by ELISA. Decitabine treatment induced robust secretion of IFN- $\beta 1$ (IFNB1) and IFN- $\lambda 1$ (IL29) (Figure 9, A and B), as well as CXCL10 and CCL5 (Figure 9, C and D). These results verify that these ccRCC cells secrete inflammatory and chemoattractant cytokines when treated with decitabine.

Next, we wanted to assess whether this increased cytokine secretion was robust enough to acutely induce inflammatory signaling in naive (resting) $\mathrm{T}$ cells. Conditioned media from decitabine-treated 786-0 cells induced antiviral signaling in naive $\mathrm{T}$ cells, although less than conditioned media obtained from 7860 cells transfected with a synthetic viral mimic (polyinosinic-polycytidylic acid; poly-IC) (Figure 9, E-J). $\mathrm{T}$ cells stimulated with conditioned media from 786-0 cells treated with $0 \mathrm{nM}$ decitabine (DMSO) showed 
A
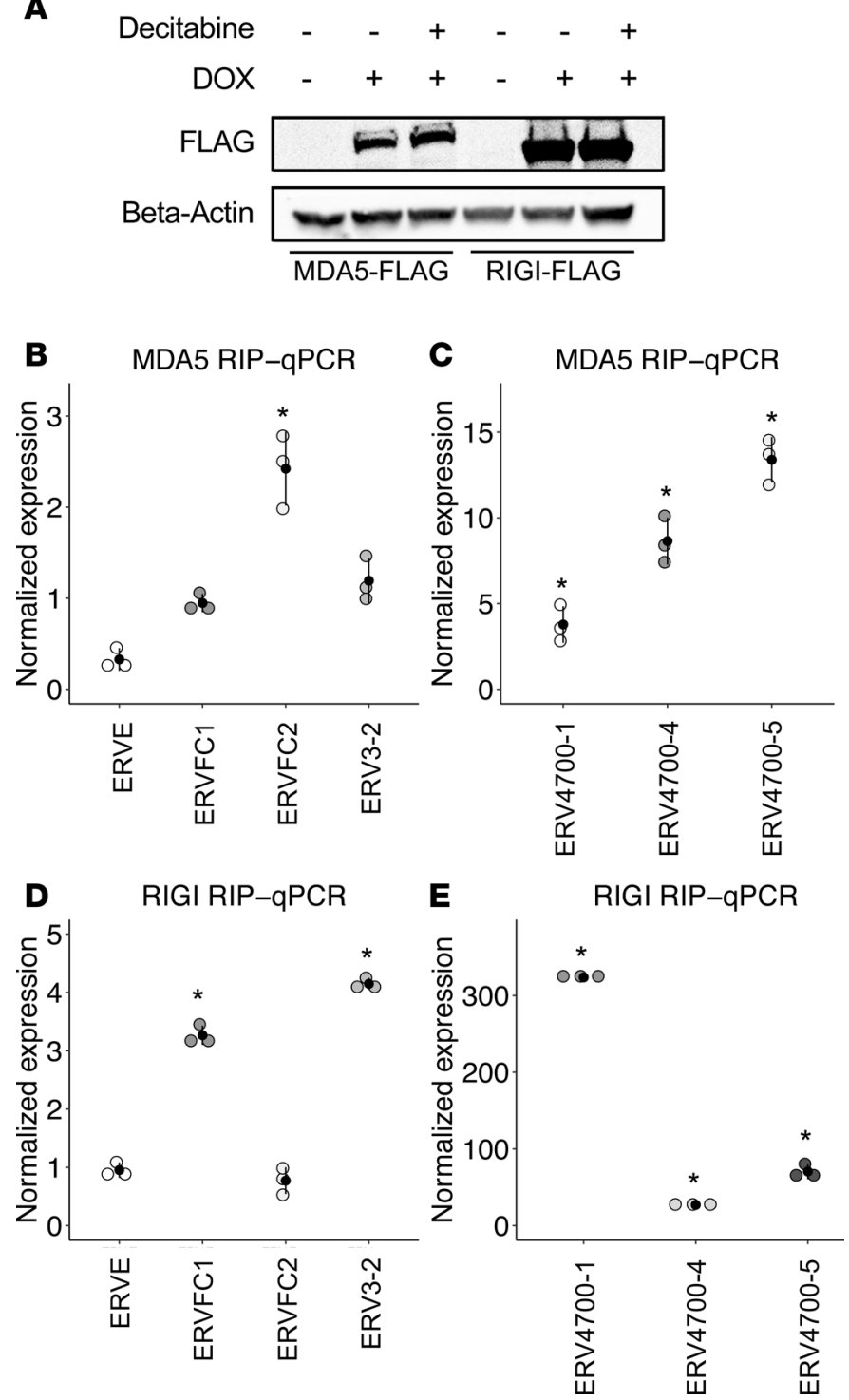

Figure 5. MDA5 and RIGI bind ERVs induced by DNA hypomethylation. (A) Verification of Doxyline (DOX) inducible MDA5-FLAG and RIGI-FLAG proteins in 786-0 cell lines by immunoblot analysis. Indicated cell lines were treated with and without DOX and decitabine. Addition of DOX induces expression of MDA5-FLAG or RIGI-FLAG constructs. MDA5FLAG and RIGI-FLAG protein was detected using anti-FLAG M2 antibody. $\beta$-Actin was used as a loading control. (B-E) Enrichment of ERVs (ERVE, ERVFC1, ERVFC2, ERV3-2, and ERV4700) bound by MDA5 (B and C) and RIGI (D and E) was assessed by RIP. IP of MDA5-FLAG and RIGI-FLAC was performed using anti-FLAG M2 antibody. ERV expression was assessed by qPCR. Values shown as fold-enrichment over input of the $100 \mathrm{nM}$ decitabine-treatment group, normalized to the $0 \mathrm{nM}$ decitabine-treatment group. Black dot and line indicate mean $\pm \mathrm{SD}(n=3)$. Significance (100 nM fold-enrichment MDA5/RIGI-FLAG IP versus $0 \mathrm{nM}$ fold-enrichment MDA5/RIGI-FLAG IP) assessed by 2-tailed $t$ test, and $P$ values were adjusted via Holm-Bonferroni correction. ${ }^{*} P<0.05$.

lower induction of antiviral signaling than fresh media. This could be due to in part to secretion of immunosuppressive factors by 786-0 cells at baseline. Thus, decitabine pretreatment of ccRCC cells could represent a potential therapeutic strategy to mitigate tumor-associated immunosuppression. To assess whether increased cytokine secretion could also enhance $\mathrm{T}$ cell activation, $\mathrm{T}$ cells were isolated from healthy donor blood and cultured in the presence of conditioned medias, and further stimulated with IL-7, only anti-CD3 antibody, or both anti-CD3/CD28 antibodies. IL-7 treatment conserves T cell viability and homeostasis without providing activating/differentiating stimulus (31) and serves as a baseline control for normalization of experimental groups. The IL-7 treatment group allowed us to assess whether conditioned media could provide sufficient stimulus for $\mathrm{T}$ cell activation. To provide a partial stimulatory signal, $\mathrm{T}$ cells were treated with 
A
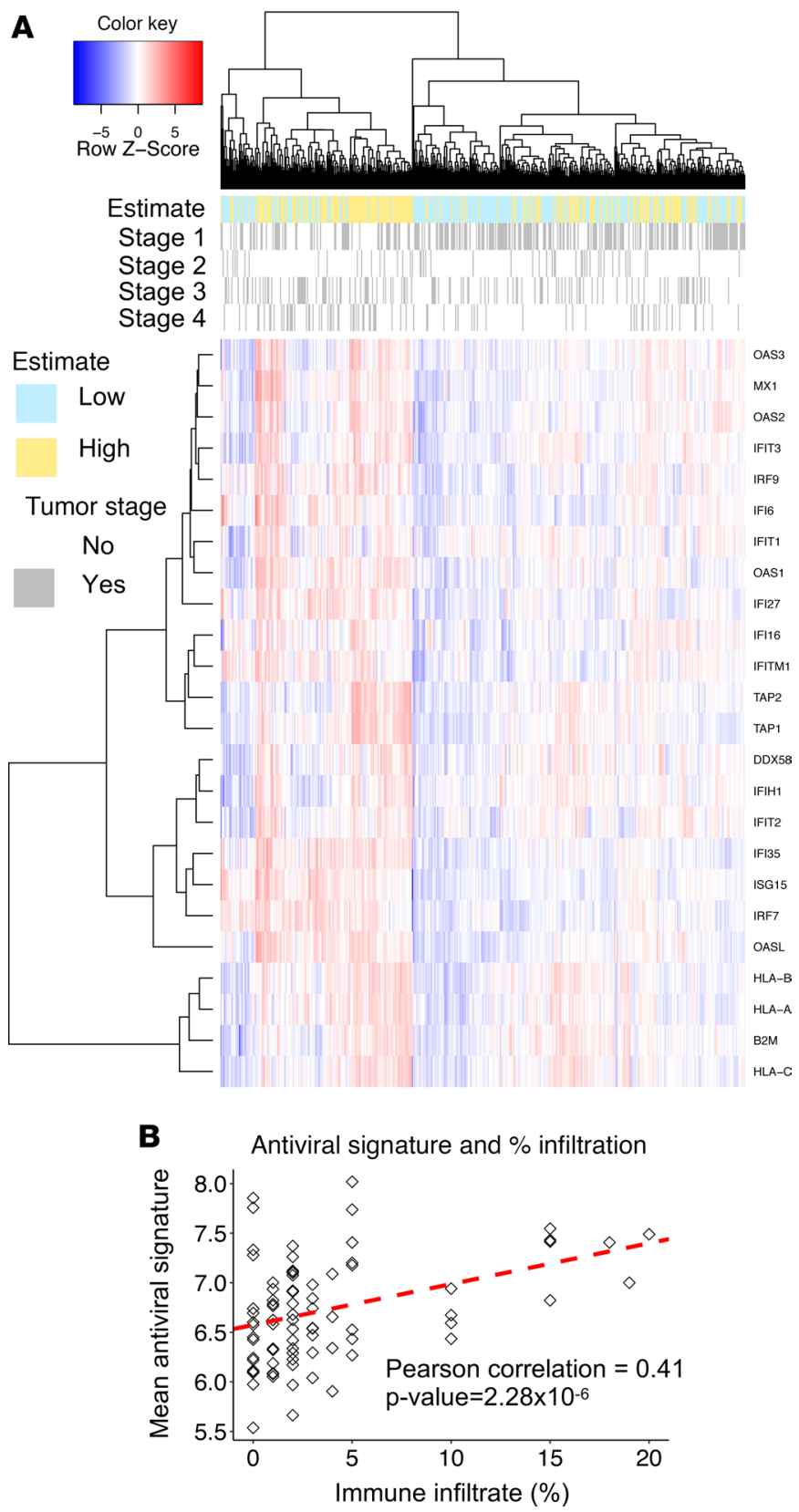

Figure 6. Antiviral response genes are expressed in ccRCC tumors with high immune cell infiltrate. (A) Heatmap for unsupervised cluster analysis was performed using antiviral response genes in ccRCC TCGA RNAseq data $(n=485)$. Barchart under dendrogram indicate ESTIMATE Immune Score and tumor stage. Color scheme indicated in key. (B) Scatterplot showing the mean of the antiviral signature and the corresponding percentage of histological immune infiltrate according to pathological evaluation in TCGA biospecimen tab. Of the 485 TCGA ccRCC samples with RNAseq data, histology immune infiltrate data were available for 126 samples. Each dot represents a sample. Red dotted line shows regression line fitted to the data (Pearson correlation $=0.41 ; P=2.28 \times 10^{-6}$ ).

anti-CD3 antibody to assess whether conditioned media could enhance activation in the absence of costimulatory stimulus. T cells were treated with both anti-CD3 and anti-CD28 antibodies to provide both TCR activation and costimulatory signals necessary for full $\mathrm{T}$ cell activation. This allowed us to assess whether conditioned media could enhance $\mathrm{T}$ cell activation in the presence of full activating stimuli.

$\mathrm{T}$ cell activation was assessed by flow cytometry after 5 days. After gating on live lymphocyte singlets, we separately gated for $\mathrm{CD} 8^{+}$and $\mathrm{CD} 4^{+}$subsets, and $\mathrm{T}$ cell activation was separately evaluated by surface expression of $\mathrm{CD} 25$ and $\mathrm{CD} 71$. When activated, both $\mathrm{CD} 4^{+}$and $\mathrm{CD} 8^{+}$subsets show increased surface expression of CD25 and CD71 (32). Consistently, CD25 and CD71 levels showed robustly increased 


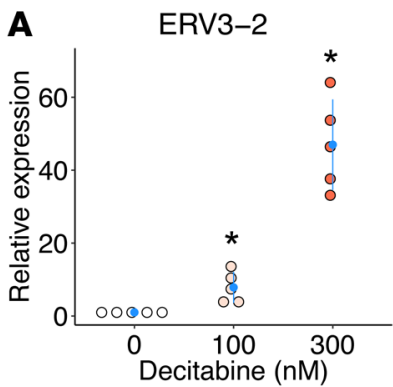

C

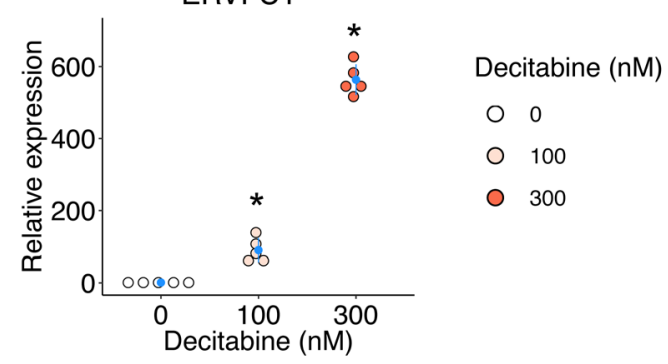

ERV4700

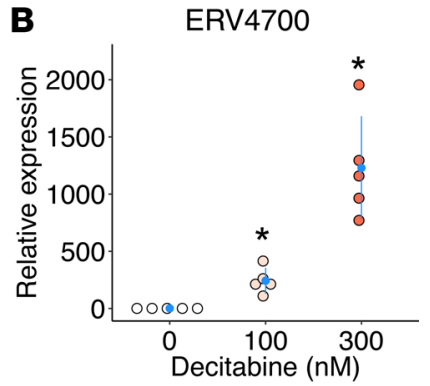

Decitabine (nM)

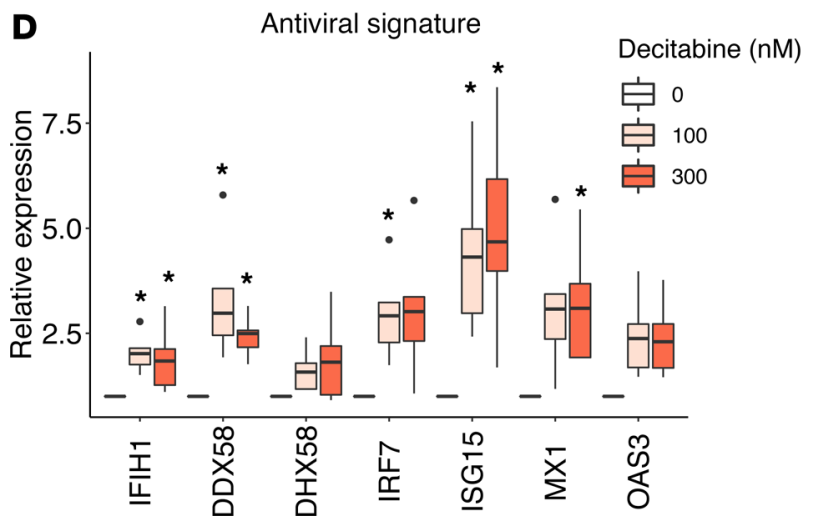

Figure 7. DNA hypomethylation in primary ccRCC cells induces ERV expression and activates antiviral signaling. Patient-derived ccRCC tumors were dissociated to establish primary cell cultures in vitro, cells were treated with the indicated doses of decitabine (or DMSO) for 3 days, and RNA was harvested on day 5 for qPCR analysis to measure expression of select ERVs (ERV3-2, ERV4700, and ERVFC1) and antiviral signaling. (A-C) Dot plots show expression of ERV3-2 (A), ERV4700 (B), and ERVFC1 (C). Each dot represents mean expression for each primary ccRCC cell line measured in triplicate. Blue dot and line indicate mean $\pm S D(n=5)$. (D) Box-and-whisker plot shows expression of antiviral signaling genes in primary ccRCC cell lines $(n=5)$ treated with indicated doses of decitabine (or DMSO vehicle) as indicated by box color in key. Box ends represent the first and third quartiles, and the horizontal line inside the box corresponds to the median. The whiskers span from minimum or maximum values, and data points outside this range correspond to outliers. Significance ( $100 \mathrm{nM}$ versus $0 \mathrm{nM} ; 300 \mathrm{nM}$ versus $0 \mathrm{nM}$ ) assessed by paired $t$ test, and $P$ values were adjusted via Holm-Bonferroni correction. ${ }^{*} P<0.05$.

expression in anti-CD3/CD28-stimulated cells. This was observed for all experimental groups of $\mathrm{T}$ cells. We observed a dose-dependent, significant increase in CD25 and CD71 expression in CD8 ${ }^{+}$(Figure 10, A and $\mathrm{B}$ ) and $\mathrm{CD}^{+}$(Figure 10, C and D) $\mathrm{T}$ cells cultured in $300 \mathrm{~nm}$ decitabine. This was comparable with the positive control poly-IC conditioned media. Finally, we confirmed that decitabine treatment would not independently impair T cell activation and was similar to those of the DMSO-treated group (Supplemental Figure 13). Thus, although not sufficient to independently activate $\mathrm{T}$ cells, conditioned media from decitabine-treated cells showed competency to augment a stimulated response.

Conditioned media from decitabine-treated $c c R C C$ cells enhances $T$ cell chemotaxis. Cytokines also have important roles in mediating immune cell recruitment to sites of inflammation. Our data show increased secretion of CXCL10 and CCL5 in ccRCC cells treated with decitabine. Given that these are important cytokines mediating $\mathrm{T}$ cell recruitment to sites of inflammation, we hypothesized that increased CXCL10 and/or CCL5 secretion could enhance T cell chemotaxis toward ccRCC cells treated with decitabine. 
A

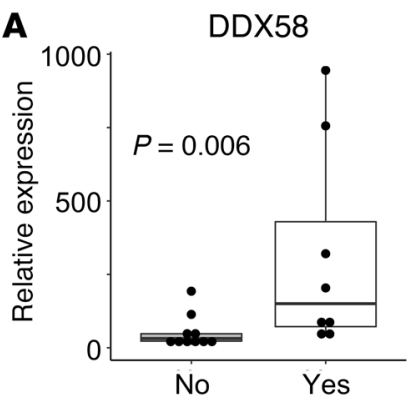

C

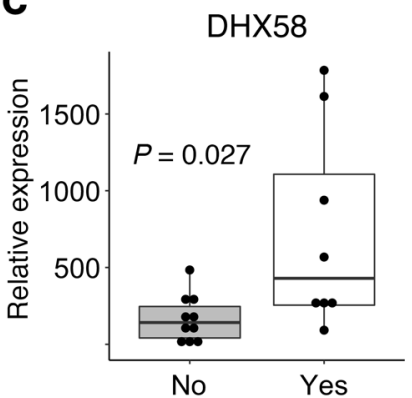

$\mathbf{E}$

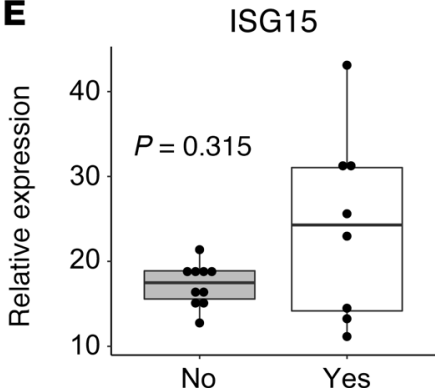

B

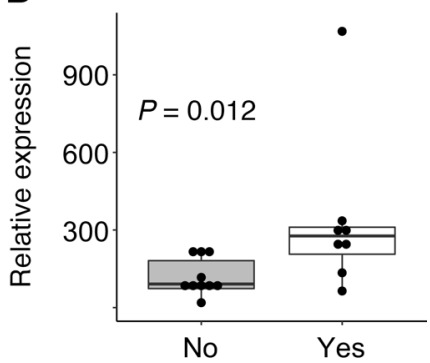

D

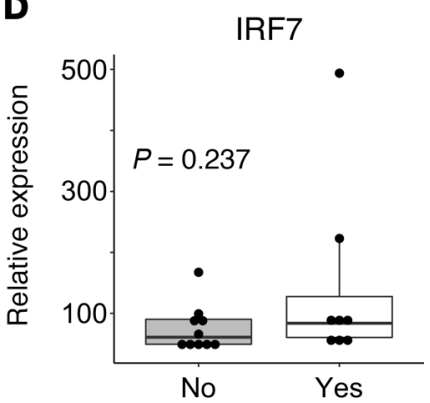

Responder

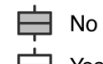

Figure 8. Activation of antiviral signaling is associated with response to ICB in ccRCC patients. Gene expression assayed by qPCR using total RNA was extracted from FFPE samples. Box-and-whisker plots show expression levels of antiviral response genes in a cohort of ccRCC patients who responded (Yes, gray shaded box; $n=13$ ) or did not respond (No, white box; $n=11$ ) to single-agent PD1 or PDL1 blockade. Overlaid dots represent expression values for individual patients. Box ends represent the first (lower) and third (upper) quartiles, and the horizontal line inside the box corresponds to the median. The whiskers span from minimum or maximum values, and data points outside this range correspond to outliers. Significance (responder versus nonresponder) assessed by Mann-Whitney $U$ test. $P$ values are indicated in plot.

We performed Transwell assays to measure migration of activated $\mathrm{T}$ cells across a membrane into the reservoirs containing conditioned media derived from 786-0 cells treated with DMSO or decitabine, as well as 786-0 cells transfected with poly-IC. T cell migration across the membrane was quantified by flow cytometry. Consistently, activated T cells migrated toward decitabine conditioned media more than DMSO conditioned media (Figure 10E). This was also true for poly-IC conditioned media. To assess if this increased migration is dependent on CCL5 and/or CXCL10 secretion, migration assays were performed with blocking antibodies for these cytokines. Migration was greatly attenuated in the presence of blocking antibody (Figure 10F). Antibody for CXCL10 decreased T cell migration slightly more than CCL 5 blocking antibody. This was also apparent in the poly-IC-treated control group. These results show that $\mathrm{T}$ cells do migrate more toward ccRCC cells treated with decitabine and suggest that CXCL10 and/ or CCL5 could, in part, mediate this effect.

\section{Discussion}

ICB for the clinical treatment of $\mathrm{ccRCC}$ has led to durable responses for some patients. However, not all patients respond to ICB, and others develop resistance to ICB. We recently reported that high ERV expression is associated with increased response to ICB in kidney cancer $(10,11)$. ERV expression can 

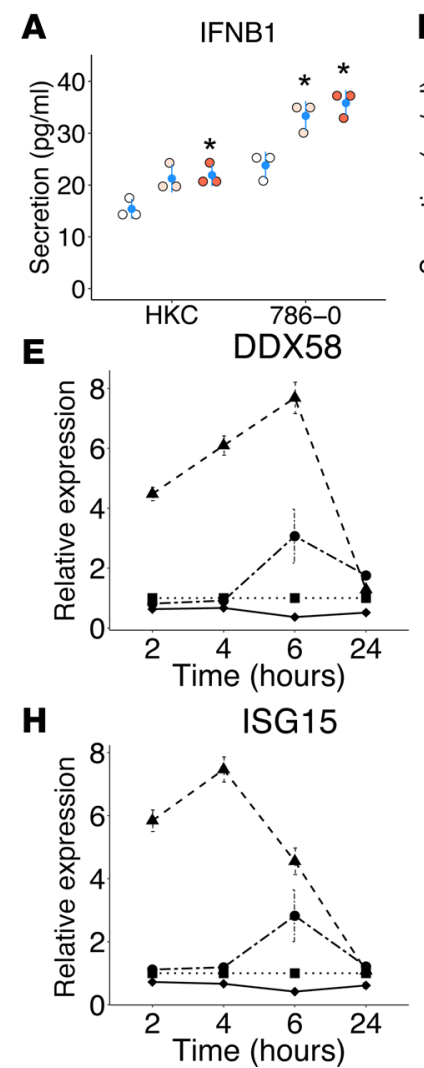
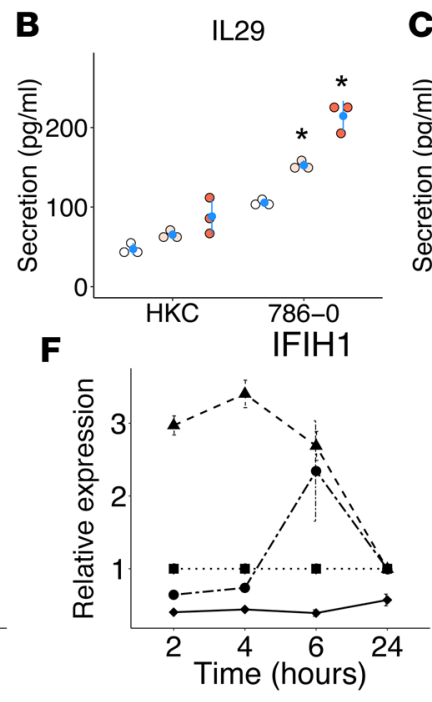

I

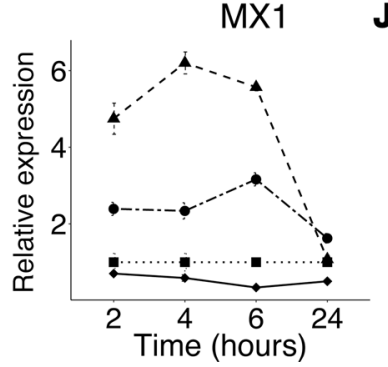

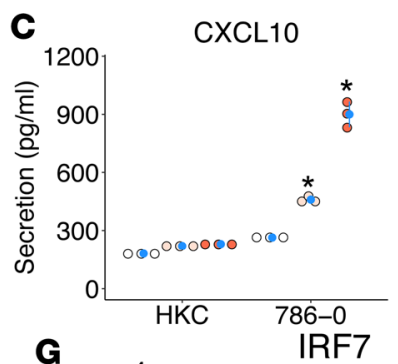
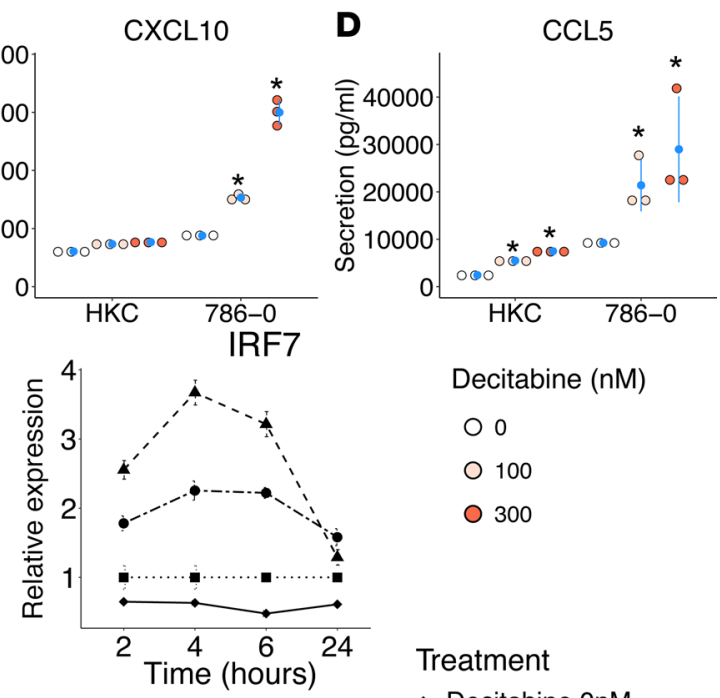

Decitabine $(\mathrm{nM})$

00

O 100

O 300

Treatment

- Decitabine OnM

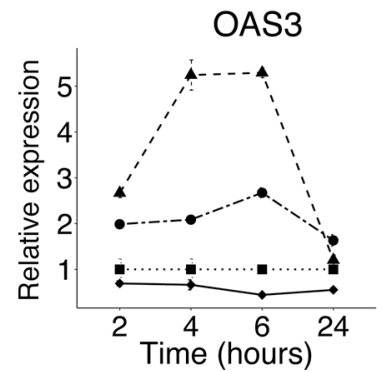

- Decitabine 300nM

- Poly-IC

- Fresh Media

Figure 9. ccRCC cells treated with decitabine secrete IFNs and inflammatory cytokines and can activate antiviral signaling in naive T cells. (A-D) HKC and 786- 0 cells were treated with indicated doses of decitabine (or DMSO) for 3 days, fresh media was add 24 hours before harvest (day 4), and conditioned media was harvested on day 5. Dot plots show levels of indicated cytokines - IFN- $\beta 1$, or IFNB1 (A); IFN- $\lambda 1$, or IL29 (B); CXCL10 (C); and CCL5 (D) were measured by ELISA in culture media on day 5. Dot plot colors indicate decitabine dose. Blue dot and line indicate mean \pm SD $(n=3)$. Significance (100 $\mathrm{nM}$ versus $0 \mathrm{nM} ; 300 \mathrm{nM}$ versus $0 \mathrm{nM}$ ) assessed by 2-tailed $t$ test, and $P$ values were adjusted via Holm-Bonferroni correction. ${ }^{*} P<0.05$. (E-J) $786-0$ cells were treated with decitabine or DMSO for 3 consecutive days; fresh media was added and harvested 24 hours later to obtain conditioned media. T cells isolated from healthy donor blood were cultured in conditioned media for 2, 4, 6, and 24 hours. Antiviral signaling was assessed measuring expression of DDX58 (E), IFIH1 (F), IRF7 (C), ISG15 (H), MX1 (I), and OAS3 (J) by qPCR.

be pharmacologically modulated in several cancer types using DNA hypomethylating agents $(17,18)$. In this study, we showed that decitabine, a DNA hypomethylating agent, could not only induce ERV expression, but also other TEs, such as LINE-1, in ccRCC. Importantly, we found decitabine induced ERV3-2 and ERV4700, which we previously reported as prognostic indicators of response to ICB in ccRCC (10, 11). While all cell lines tested showed increased TE expression and activation of antiviral responses, this effect seemed to be attenuated in the 2 noncancerous transformed kidney cell lines included in this study. Our results suggest that cancerous cells might be more sensitive to epigenetic modulation of TEs and activation of antiviral signaling than noncancerous transformed kidney cells, and this is worthy of further investigation. This increased sensitivity of cancerous cells to epigenetic modulation of TE expression could be attributed to alterations accumulated in cancer cells that whither at epigenetic or other regulatory safeguards, which can mitigate the effect of TE expression and activation antiviral signaling. For instance, increased TE expression was reported by loss of $\operatorname{VHL}(33,34)$, which is the most common alteration in kidney cancers $(24,25)$. All 3 ccRCC cell lines used herein have mutations in $V H L$. These results suggest that activation of TE expression by DNA hypomethylating agents, such as decitabine, could be partially selective to ccRCC cancer cells that possess genetic abnormalities not present in normal kidney cells.

TE/ERV expression produces abnormal or unusual RNA species that can form double-stranded structures by virtue of their repetitive nature. These RNAs can activate pattern-recognition receptors, namely RIGI (DDX58), MDA5 (IFIH1), and TLR3. Upon binding double-stranded RNA (dsRNA), RIGI and MDA5 initiate cellular antiviral signaling, culminating with activation of IFN and NF-kB signaling (35). In agreement with previous reports $(17,18)$, our data show that decitabine-induced TE expression was accompanied by activation of antiviral signaling, as demonstrated by robust upregulation of DDX58 and 
A $\quad$ CD25 in CD8+ T-cells

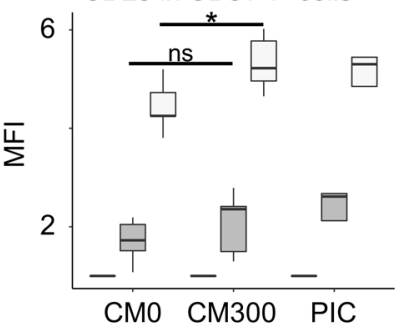

B
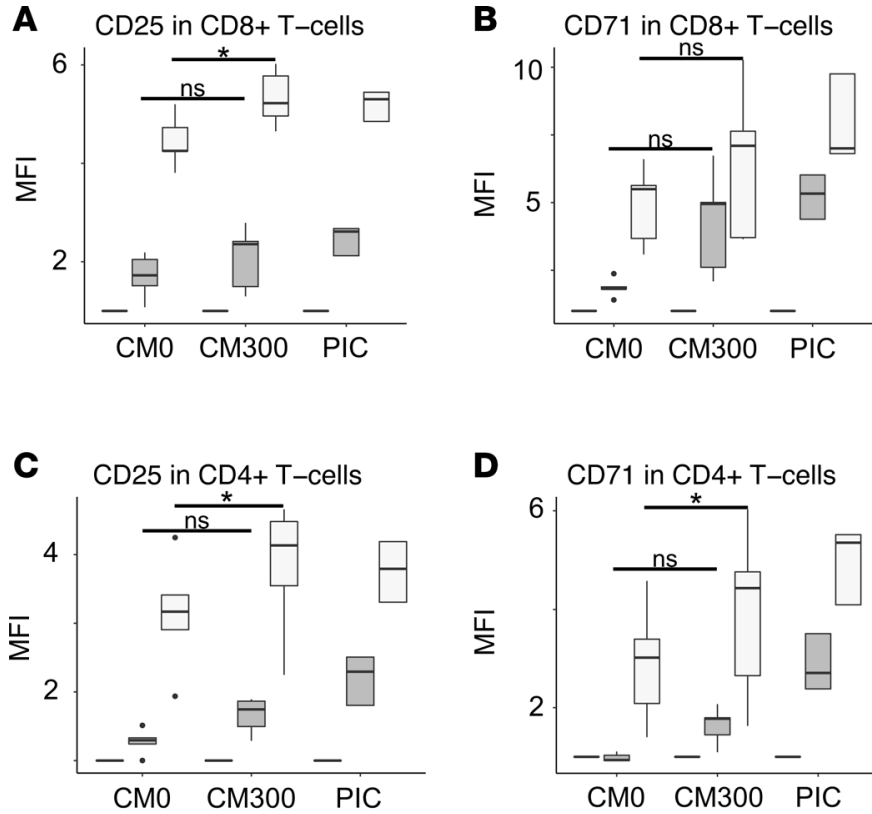

D

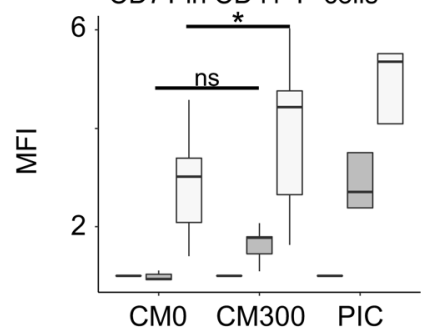

E
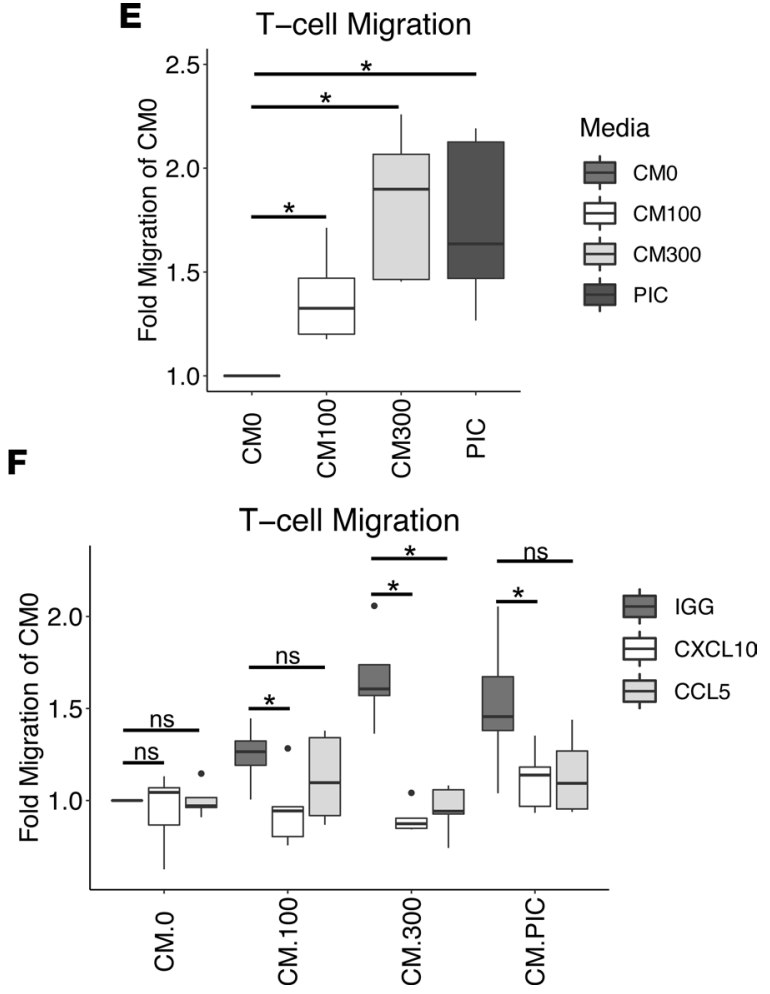

Figure 10. Conditioned media from ccRCC cells treated with decitabine can enhance T cell function. 786-0 cells were treated with decitabine or DMSO for 3 consecutive days; fresh media was added and harvested 24 hours later to obtain conditioned media. Conditioned media from 786-0 cells transfected with poly-IC was used as a positive control. Healthy donor T cells $(n=5)$ were cultured in conditioned media, and T cell function was assessed by flow cytometry. (A-D) T cells were stimulated with IL7, anti-CD3 antibody, and anti-CD3/CD28 antibodies and cultured for 5 days. Cell size, CD4, CD8, CD25, and CD71 was measured by flow cytometry to evaluate T cell activation. Surface expression of CD25 and CD71 (as median fluorescence intensity; MFI) was measured in CD8 ${ }^{+}$ $(\mathbf{A}$ and $\mathbf{B})$ and $\mathrm{CD}^{+}(\mathbf{C}$ and $\mathbf{D})$ T cell subsets. ( $\mathbf{E}$ and $\mathbf{F}$ ) Migration of healthy donor T cells $(n=5)$ activated using anti-CD3/CD28 antibodies was assessed by Transwell assay. (E) Migration of activated T cells toward indicated conditioned medias. (F) Migration of activated T cells toward the indicated conditioned medias treated with either ICG isotype antibody, CXCL10 antibody, or CCL5 antibody $(n=5)$. Box-and-whisker plots show expression antiviral signaling genes in primary ccRCC cell lines $(n=5)$ treated with indicated doses of decitabine (or DMSO vehicle) as indicated by box color in key. Box ends represent the first and third quartiles, and the horizontal line inside the box corresponds to the median. The whiskers span from minimum or maximum values, and data points outside this range correspond to outliers. Significance assessed by paired $t$ test, and $P$ values were adjusted via Holm-Bonferroni correction. ${ }^{*} P<0.05$.

IFIH1, as well as downstream effectors (IRF7, ISG15, MX1, and OAS3). Our results show that KO of RIGI, MDA5, and MAVS attenuated antiviral signaling, which implicates ERV species induced by decitabine in this process. It is important to note that decitabine resulted in widespread DNA hypomethylation throughout the genome and was not just restricted to TEs. Thus, it is likely that this DNA hypomethylation contributes to activation of antiviral signaling. Although off-target effects of hypomethylation may play a role in decitabine's effects, treatment results in activation of innate immune responses that conveys the potential to increase immunogenicity and promote rejection by the immune system.

We showed enrichment of several decitabine-induced ERVs in RIGI and MDA5 IPs. Enrichment of decitabine-induced ERVs in RIGI/MDA5 IPs was species dependent, which was expected, as MDA5 and RIGI recognize distinct chemical and structural features of RNAs. Involvement of RIGI in this process was not expected, since RIGI senses short 5'-tri/diphosphorylated dsRNA generally absent from the host transcriptome, although direct interactions between dsRNAs and RIGI have been reported (36). Additionally, RIGI is important for antitumor immune responses associated with radiotherapy whereby radiation induces noncoding RNAs (ncRNAs) that are sensed by RIGI to activate immune signaling (37). In this regard, many ncRNAs are believed to have originated through evolutionary exaptation of TEs and ERVs (38). Further studies are warranted to characterize the full repertoire of decitabine-induced ERV/dsRNAs bound by RIGI and MDA5.

As part of the activation of innate antiviral responses, we showed that decitabine induced secretion of IFNs (IFNB1, IL29) and cytokines (CXCL10, CCL5), which we hypothesized could enhance T cell function. Here, we show that factors secreted by ccRCC cells treated with decitabine are capable of activating antiviral signaling in naive (resting) $\mathrm{T}$ cells. Next, we performed in vitro $\mathrm{T}$ cell activation assays to 
determine whether this increased secretion could enhance $\mathrm{T}$ cell activation. Although enhanced de novo T cell activation with decitabine or poly-IC conditioned media was not observed, T cell-stimulated activity was enhanced compared with the DMSO conditioned media control group. We showed that increased levels of CXCL10 and CCL5 secreted by decitabine-treated cCRCC cells significantly increased T cell chemotaxis in Transwell migration assays, which was attenuated by blocking antibodies against CXCL10 and CCL5. Increased levels of CXCL10 and CXCL9 are associated with increased CD8 ${ }^{+} \mathrm{T}$ cell infiltration in tumors (39), which has been shown to be associated with response to anti-PD1 therapy (40). Thus, our results strongly suggest that activation of TEs and antiviral responses induced by decitabine could contribute to immunogenicity by enhancing T cell migration toward ccRCCs through increased IFN and cytokine (CXCL10 and CCL5) secretion.

Our data suggest that epigenetic modulation of TE expression could be an effective strategy to enhance response to ICB in ccRCC. Using RNAseq, we were able to identify a number of genes induced by decitabine in ccRCC cells, many of which have immune-related functions, including NF- $\kappa \mathrm{B}$ and IFN signaling. Further analyses using TCGA ccRCC RNAseq data (25) identified a number of significant associations between these decitabine-induced genes and immune infiltrate. In an independent gene expression data set (30), we confirmed significant associations between immune cell infiltrate in ccRCCs for $\sim 70 \%$ of these genes. In fact, using just 24 antiviral response genes, we were able to achieve remarkable separation of TCGA samples according to the level of immune infiltrate. Furthermore, we show that ERV expression and activation of antiviral signaling could also be achieved in patient-derived primary ccRCC cultures. Finally, we confirmed significant upregulation of DDX58, IFIH1, and DHX58 in another independent cohort of ccRCC patients who responded to single-agent anti-PD1 therapy. Several clinical trials combining hypomethylating agents with ICB are underway (ClinicalTrials.gov NCT02397720, NCT03825367, NCT03094637, NCT02260440, NCT03233724, NCT02775903, NCT02811497), and future studies should interrogate mechanisms of enhanced immune response in order to optimize this opportunity to bring durable immunologic response to patients with cancer.

\section{Methods}

Reagents. Stock solutions of decitabine (Selleck Chem) were prepared in DMSO at $50 \mathrm{mM}$. Poly-IC (MilliporeSigma) was prepared in distilled water at $1 \mathrm{mg} / \mathrm{mL}$. Double-stranded poly-IC molecules were prepared in accordance to manufacturer recommendations. Stock solutions were stored at $-80^{\circ} \mathrm{C}$ in aliquots. A fresh aliquot was used for each drug treatment.

Patient samples. Formalin-fixed, paraffin-embedded (FFPE) metastatic ccRCC samples were collected at the Vanderbilt-Ingram Cancer Center using an IRB-exempt waiver of consent for FFPE (approved by the Vanderbilt University Medical Center Human Research Protection Program). Samples collected by the Rutgers Cancer Institute of New Jersey were collected with approval from the Rutgers Institutional Review Board.

All tissues were evaluated by a pathologist using H\&E staining, confirming clear cell histology, and only samples containing $\geq 70 \%$ tumor cells were included. The cohort from patients treated with single-agent PD1/PDL1 blockade included 13 patients with partial response and progression-free survival of at least 6 months, as well as 11 patients who demonstrated immediate progressive disease (10). Clinical and demographic data for patient cohort are available in Supplemental Table 9.

Fresh ccRCC tissue was collected from 5 patients and processed by mechanical dissociation and enzymatic digestion. Cells were cultured in DMEM (Thermo Fisher Scientific, catalog 11965092) supplemented with 5\% FBS (Gemini Bio-Products, catalog 100-106), 1\% penicillin-streptomycin (Thermo Fisher Scientific, catalog 15140122), and 1\% L-glutamine (Thermo Fisher Scientific, catalog 25030081) for 3 to 5 days before beginning drug treatments.

Cell lines. All cell lines used were human. 786-0, A498, and UMRC2 ccRCC cell lines were purchased from ATCC (American Type Culture Collection). Human RPTec noncancerous transformed kidney cells were purchased from ATCC, while the HKC cell line was a gift from Lorraine Claire Racusen (Johns Hopkins University School of Medicine, Baltimore, Maryland, USA) (41). Monthly Mycoplasma testing using the ATCC Universal Myco Detection Kit verified that all cell line were Mycoplasma free. Cell line were cultured for a maximum of 15 passages. The 786-0 cells were cultured in RPMI (Corning, catalog 10-040-CV), while A498, UMRC2, and HKC cells were cultured in DMEM (Thermo Fisher Scientific, catalog 11965092). Media was supplemented with 10\% FBS (Gemini Bio-Products, catalog 100-106), 1\% penicillin-streptomycin (Thermo Fisher Scientific, catalog 15140122), and 1× 
L-glutamine (Thermo Fisher Scientific, catalog 25030081). RPTec cells were cultured in REBM Basal Medium (Lonza, CC-3191) supplemented with REGM SingleQuots supplements (Lonza, CC-4127).

Treatments. Cells were treated daily with decitabine (100 nM and $300 \mathrm{nM})$ or DMSO for 3 consecutive days. After 72 hours, fresh media was added; cells were cultured for 2 additional days and harvested at 120 hours (day 5). Mock-treated cells were treated with DMSO for 3 days (days 0, 1, and 2), and protein was harvested at day 5 , unless specified otherwise. For conditioned media, cells were treated with indicated doses of decitabine for 3 days, after which, fresh media was added and cells were cultured for an additional 24 hours before harvesting media. For poly-IC positive control, cells were transfected with poly-IC ( $50 \mathrm{nM})$ using FuGENE-6 reagent for 4 hours in serum-free media (Optimem). After 4 hours, cells were washed, 20 and fresh media was added. Conditioned media was centrifuged $(400 \times g)$ at $4^{\circ} \mathrm{C}$ for 10 minutes to pellet cellular debris and filter sterilized $(40 \mu \mathrm{m})$. These treatment schedules were maintained for all experiments, unless stated otherwise.

Cellular viability assay. Cells were seeded in a white flat-bottom 96-well plate (Greiner, catalog 655075) at a density of 800 cells/well and allowed to adhere overnight. Cells were treated daily with decitabine $(0$, $10,50,100,250,500,1000,5000 \mathrm{nM}$ ) for 3 days and cellular viability was assessed using the CellTiter-Glo Luminescent Cell Viability Assay (Promega) in accordance with the manufacture's recommendations.

DNA samples. DNA was extracted from cell lines using the DNeasy Blood and Tissue Kit (QIAGEN). DNA was treated with RNAse A, DNase, and protease-free (Thermo Fisher Scientific). DNA concentration was determined using a NanoDrop2000 Spectrometer, and DNA integrity was assessed by agarose gel electrophoresis.

$R N A$ samples. RNA was extracted using the miRNeasy Mini Kit (QIAGEN). DNase treatment was performed during extraction using the on-column method in accordance to the manufacturer's specifications. For qPCR, RNA quality and concentration were assessed using a NanoDrop ND-1000 spectrophotometer (NanoDrop Technologies). For RNAseq, RNA quality was determined using an Agilent BioAnalyzer, and only RNAs with an RNA integrity number score greater than 7 were sequenced.

Protein extraction and immunoblot analysis. Whole cell lysate was extracted using radioimmunoprecipitation assay buffer supplemented with $1 \times$ Halt protease and phosphatase inhibitors (Thermo Fisher Scientific). For Western blot analysis, $40 \mu \mathrm{g}$ of protein was loaded for each sample. Antibodies used included rabbit anti-DNMT1 (1:1000; clone D63A6 XP mAb, Cell Signaling Technology, 5032), -IRF7 (1:1000; Cell Signaling Technology, 4920), -RIGI (1:500; clone D33H10, Cell Signaling Technology, 4200), -MDA5 (1:500; clone D10F10, Cell Signaling Technology, 5321) and - $\beta$-actin (1:10000; Abcam, ab8227), and mouse antiISG15 mAb (1:500; clone F-9, Santa Cruz Biotechnology sc-166755). Mouse monoclonal anti-FLAG antibody (1:1000; clone M2, MilliporeSigma, F1804) was used for verification of FLAG-tagged MDA5 and RIGI. $\beta$-Actin was used for loading control.

$q P C R$. First-strand cDNA synthesis was performed using $250 \mathrm{ng}$ total RNA, random hexamers, and the SuperScript IV Reverse Transcriptase Kit (Invitrogen). qPCR was performed on a CFX96 Touch Real-Time PCR Detection System (Bio-Rad) using the SYBR green master mix reagent (Invitrogen). qPCR primers were designed using PrimerBank (42). Primer sequences that were used for qPCR are provided in Supplemental Table 10. ERV4700 was quantified using the $2 \times$ TaqMan Universal PCR Master Mix, as previously described (11). All analyses were performed in triplicate, and relative RNA levels were determined using HPRT1 as an endogenous control. A HeLa control RNA sample was included for interplate calibration. Relative gene expression levels were calculated using the $2^{-\Delta \Delta \mathrm{Ct}}$ method.

DNA methylation assay. Genomic DNA (250-750 ng) was treated with sodium bisulphite using the EZ DNA methylation kit (Zymo Research). DNA methylation was quantified using the Illumina Infinium HumanMethylationEPIC (EPIC) BeadChip run on an Illumina iScan System using the manufacturer's standard protocol. Raw IDAT files were processed with Illumina's GenomeStudio software V2011.1 and background normalized using negative control probes to generate methylation $\beta$ values, which were used for all downstream analyses. We used MethylationEPIC_v-1-0_B2 manifest for processing EPIC data. EPIC data files are available from NCBI Gene Expression Omnibus (GEO; https://www.ncbi.nlm.nih. gov/geo) under accession number GSE146179.

RNAseq. RNAseq was performed by the Vanderbilt Technologies for Advanced Genomics (VANTAGE) Sequencing Core at Vanderbilt University. RNASeq libraries were prepared using $350 \mathrm{ng}$ of total RNA and the NEBNext Ultra II RNA Library Prep (NEB, E7765S) per manufacturer's instructions, with mRNA enriched via poly-A selection using Oligo(dT) beads. The RNA was then thermally fragmented and converted to CDNA, adenylated for adaptor ligation, and PCR amplified. The libraries were sequenced 
using the NovaSeq 6000 with 150 bp paired end reads. RTA (version 2.4.11; Illumina) was used for base calling, and analysis was completed using MultiQC v1.7.

For analysis of canonical gene expression, sequencing reads were aligned against the human GENCODE genome, hg38/GRCh38 (Version 28), using the Spliced Transcripts Alignment to a Reference (STAR) software (43) using default parameters. For the analysis of TEs, reads were mapped to the human genome (CRCh38/hg38) using the STAR aligner (43), with the following options: --runRNGseed 123 --outSAMmultNmax 1 --outFilterType BySJout --alignSJoverhangMin 8 --alignSJDBoverhangMin 1 --outFilterMismatchNmax 15 --alignIntronMin 20 --alignIntronMax 1000000 --alignMatesGapMax 1000000 --outFilterMultimapNmax 100 --winAnchorMultimapNmax 100 --outMultimapperOrder Random. For analysis of TE expression, we allowed for multimapping alignments whereby the STAR aligner randomizes the choice of the primary alignment from the highest-scoring alignments. Mapped reads were annotated and quantified using featureCounts (44). Gene transfer format (GTF) files for gene annotation were downloaded from GENCODE (Version 28, hg38/GRCh38). Gene transfer format (GTF) file (GRCh38_ rmsk_TE.gtf) for TE annotations were downloaded from the TEtranscripts website (http://hammelllab. labsites.cshl.edu/software/\#TEtranscripts) (45). Normalization and differential expression was performed using DESeq2 (46). Genes showing FDR $<0.05$ and $\log _{2}$ difference greater than 1.5 in magnitude were considered differentially expressed in pair-wise comparisons. For TE expression, TEs with an FDR $<0.05$ (ANOVA) and $\log _{2}$ difference greater than 0.75 in magnitude were considered differentially expressed in pair-wise comparisons. The top most significantly differentially expressed genes were considered for subsequent functional enrichment using Gene Set Enrichment Analysis (47, 48). Gene expression RNAseq data files are available from NCBI GEO (https://www.ncbi.nlm.nih.gov/geo) under accession number GSE142381. TE expression data are provided in Supplemental Table 1.

Integration of DNA methylation and TE expression levels. Integrative analysis was performed using the 119 TEs differentially expressed between 786-0 cells treated with decitabine and DMSO vehicle. Using the TE annotations (GTF) used for the RNAseq analysis, we defined promoter regions as 2000 bases upstream and 400 bases downstream for the genomic coordinates mapped to these TEs, including those mapping to multiple locations. Before integrative analyses, the EPIC DNA methylation data were processed such that probe location was lifted over to the GRCh38/hg38 human genome assembly. For genomic integration between these potential TE promoters and the EPIC DNA methylation data, only EPIC probes overlapping with TE promoters and showing a difference of at least 30\% DNA methylation were considered $(n=92)$. DNA methylation $\beta$ values were converted to $M$ values prior to corelative analyses with matched RNAseq data. We assessed the association between TE expression and DNA methylation in 786-0 cells treated with decitabine (300 nM) or DMSO (0 nM) using Pearson's product moment correlation coefficient.

NanoString. To measure gene expression changes in immune-related pathway genes in kidney cells treated with $300 \mathrm{nM}$ decitabine or DMSO, we used the Pan-cancer immune NanoString gene panel. A total of $150 \mathrm{ng}$ of total RNA from each sample was subjected to NanoString analysis, using the Pan-cancer immune gene panel with appropriate housekeeping controls (NanoString). The NanoString assay was performed according to manufacturer recommendations. The nSolver Analysis Software (version 4.0) was used for analysis. Briefly, raw NanoString counts were initially subjected to normalization for all target RNAs in all samples based on built-in positive controls, which accounts for intersample and experimental variation. Afterward, the housekeeping genes were used for mRNA content normalization. NanoString data files are available upon request. Processed and normalized NanoString count data are provided in Supplemental Table 8.

$M A V$-, MDA5-, and RIGI-KO cells. Following puromycin selection, single cell clones were grown in 48-well plates. CRISPR/Cas9-induced mutations were identified by isolation of genomic DNA and PCR of the genomic region where the guide RNA is targeted. TOPO cloning, and Sanger sequencing of the PCR product. MAVS-, MDA5-, or RIGI-KO was verified by immunoblot of the respective protein.

RNA IP followed by quantitative PCR (RIP-qPCR). RIP was performed for FLAG-tagged RIGI and MDA5 proteins using an anti-FLAG antibody to isolate bound RNA species, as previous described (49). Isolated RNAs were analyzed by qPCR. Briefly, RIGI and MDA5 were amplified from pEF-BOS-RIGI and pEF-BOS-MDA5, cloned into pDONOR221, and recombined with pLenti-CMVtight-FL-HA-DESTBlast (Addgene) with Gateway cloning (Invitrogen) (49). Lentivirus was prepared in HEK293-FT cells. Nine cells were transfected at 50\% to $60 \%$ confluence with RIGI-FLAG, MDA5-FLAG, or empty destination vectors psPAX2 (Addgene) and pMD2.G (Addgene) using $8 \mu \mathrm{g} / \mathrm{mL}$ polybrene (MilliporeSigma). 
Seventy-two hours after transfection, the supernatant was collected, and target cells were infected at 1000 $g$ for 24 hours. Cells were selected for 2 weeks in media containing $5 \mu \mathrm{g} / \mathrm{mL}$ blasticidin (Invivogen).

Doxyline-inducible RIGI-FLAG- and MDA5-FLAG-expressing 786-0 cells were treated daily with $100 \mathrm{nM}$ decitabine or DMSO for 3 days. On day 2 of decitabine treatment, cells were treated daily with Doxyline. After applying Doxyline for 2 days, RIP was performed for FLAG-tagged RIGI and MDA5 proteins using an anti-FLAG antibody (clone M2, MilliporeSigma, M8823) to isolate bound RNA species, as previous described (50). Briefly, $1.5 \times 10^{7}$ cells were cross-linked in $1 \%$ formaldehyde for 10 minutes and subsequently quenched with $0.25 \mathrm{M}$ glycine. Cells were lysed in IP lysis buffer (50 mM HEPES [pH 7.5], 0.4 M NaCl, 1 mM EDTA, 1 mM DTT, 0.5\% TritonX-100, and 10\% Glycerol) supplemented with phenylmethylsulfonyl fluoride, complete protease inhibitor and RNase inhibitor (Invitrogen). Cells were sonicated using a Bioruptor (10 cycles, 30 seconds on, 30 seconds off). Cellular debris was removed by centrifugation at $400 \times g$ for 10 minutes at $4^{\circ} \mathrm{C}$, and $5 \%$ of the lysate was taken as INPUT. RIGI-FLAG and MDA5FLAG were immunoprecipitated using anti-FLAG Magnetic Beads (clone M2, MilliporeSigma, M8823). Beads were magnetically collected and washed 5 times with IP lysis buffer. Samples were incubated at $70^{\circ} \mathrm{C}$ for 1 hour to reverse the cross-links on nucleic acids, and RNA was extracted using Trizol reagent (Invitrogen). Extracted RNA was treated with DNase I recombinant, RNase-free (QIAGEN). cDNA was synthesized using 100 ng of RNA, as described above. Sequences for qPCR primers can be found in Supplemental Table 9. For this assay, ERV4700 was quantified using 3 different primer/probe sets corresponding to different regions of this ERV. Primer/probes ERV4700-1, ERV4700-4, and ERV4700-5 corresponded respectively to Gag 1, Pol 2, and Env 1 regions of ERV4700.

ELISA. For IFNB1, we used the IFN- $\beta$ Human ELISA Kit from Thermo Fisher Scientific (catalog 414101). For IL29, CXCL10, and CCL5 determination, we used the Quantikine HS ELISA kit (R\&D Systems). All ELISAs were performed following the manufacturer's protocol. Absorbance was measured at $450 \mathrm{nM}$, with wavelength correction at $570 \mathrm{nM}$. Samples were assayed in triplicate.

$T$ cell activation assay. Peripheral blood mononuclear cells (PBMCs) were obtained from healthy donor blood (Gulf Coast Regional Blood Center) by Ficoll-Paque (GE Healthcare) gradient centrifugation using the SepMate PBMC Isolation kit (Stemcell Technologies). The initial centrifugation was performed at $2000 \times g$ for 10 minutes at room temperature to isolate the buffy coat, and then subsequent centrifugation at $300 \times g$ for 8 minutes at room tempature to separate the PBMCs from plasma. For activation experiments, T cells were seeded at an initial density of $2 \times 10^{6}$ cells $/ \mathrm{mL}$ using conditioned media from ccRCC cells treated with decitabine or DMSO, or transfected with poly-IC. T cells were also treated with decitabine. T cells were further stimulated with either IL-7 (1:1000; Biological Research Branch, NCI, NSG number 644800), anti-CD3 antibody (1:1000; clone UCHT1, eBioscience, 14-0038-82), or anti-CD28 antibody (1:1000; clone CD28.2, eBioscience, 16-0289-81). T cell activation was assessed by flow cytometry to measure surface marker expression of CD4 (1:400; VioGreen, clone M-T466; Miltenyi Biotec, 130-113. 259), CD8A (1:400; eFluor450, clone SK1; Invitrogen, 48-0087-42), CD25 (1:400; PerCP-Cyanine5.5, clone BC96; Invitrogen, 45-0259-42), and CD71 (1:400; APC, clone OKT9; Invitrogen, 17-0719-42).

T cell migration assay. T cells were isolated and activated using both anti-CD3 and anti-CD28 antibodies, as described, in fresh $\mathrm{T}$ cell media (not conditioned media) for 5 days. On day 5 , $\mathrm{T}$ cells were plated in a 24-well Transwell plate ( 5 micron pore) at a density of $5 \times 10^{5}$ cells/well. Conditioned media was added to the lower wells. CXCL10 (RND Biosystems, MAB266) or CCL5 (RND Biosystems, MAB278) blocking antibodies were added 30 minutes prior at a final concentration of $1 \mu \mathrm{g} / \mathrm{mL}$. T cells were cultured for 90 minutes, and cellular migration into the lower reservoir was quantified by flow cytometry.

Genomic data / public data. For the TCGA ccRCC RNAseq data, raw count data for each sample included was obtained from NCBI GEO (https://www.ncbi.nlm.nih.gov/geo) under accession number GSE62944. The ESTIMATE scores for the cCRCC TCGA data were obtained from the ESTIMATE website (Verhaak Lab; https://bioinformatics.mdanderson.org/estimate) (29). The ccRCC gene expression data used for validation cohort was obtained from ArrayExpress (https://www.ebi.ac.uk/arrayexpress) under accession number E-MTAB-1980 (30). Histological immune infiltrate percentages were obtained from the KIRC TCGA biospecimen table (25). For RNA-Seq expression analysis, reads per kilobase per million (RPKM) values were calculated from RNAseq raw counts and upper quantile normalized. The ccRCC gene expression array data were upper quantile normalized.

Statistics. All bioinformatic and statistical analyses were performed in R open source programming language. Associations between decitabine-induced genes and immune cell infiltrate were identified 
using univariate linear models, and $P$ values were adjusted for multiple testing (FDR). Associations with FDR $<0.05$ were considered significant. Immune cell infiltrate was calculated using the Immune Score from the ESTIMATE algorithm (29). Two-tailed $t$ tests were used to assess significance of qPCR measurements. Two-tailed paired $t$ test were used to assess significance of ccRCC patient samples and T cell experiments. Mann-Whitney $U$ tests were used to assess significance of antiviral gene expression in a cohort of ccRCC patients treated with anti-PD1/PDL1. The Wald test was used to assess significance of differential expression analysis of RNA-Seq data. In the case of parallel test, $P$ values were adjusted via Holm-Bonferroni correction.

Study approval. Work in this study was performed with the approval of the Vanderbilt University Medical Center IRB. Informed consent was deemed unnecessary by the IRB of the Vanderbilt University Medical Center Human Research Protection Program. Tissue were collected using an IRB-exempt waiver of consent, approved by the Vanderbilt University Medical Center Human Research Protection Program and the Rutgers University Institutional Review Board.

\section{Author contributions}

The study was designed AADC, WKR, SG, and GB. Experiments were conducted by AADC, AZ, and $\mathrm{AB}$. Data were acquired by AADC and AP. Data were analyzed by AADC and AP. Reagents were provided by AADC, WD, RAH, KEB, and JK. The manuscript was written by AADC, WD, AZ, RAH, AB, AP, $\mathrm{KEB}, \mathrm{GB}, \mathrm{SG}, \mathrm{JK}$, and WKR.

\section{Acknowledgments}

ccRCC tissues were collected by the Cooperative Human Tissue Network Core at Vanderbilt University Medical Center. RNAseq was performed by VANTAGE Core at Vanderbilt University, and with support from the Vanderbilt Ingram Cancer Center. WKR is supported by R01CA198482. AAD is supported by a K01 NCI Mentored Research Scientist Development Award to Promote Diversity (1K01CA245231-01) and received previous support from an American Cancer Society Postdoctoral Fellowship Award (132921-PF-18-183-01-TBG) and T32CA009582-28. KEB is supported on K12CA090625. JK is supported by R01AI141448 and the Pew Charitable Trusts as a Pew Biomedical Scholar. GB is partly supported by an ORIEN grant (no. 826489) and a Department of Defense KRCP grant (KC180159). AP is supported by a postdoctoral fellowship from the New Jersey Comission on Cancer Research (NJCCR). SG is supported by NCI (R01CA2435478, R01CA20275200), Department of Defense (KC180159), BCRF, Hugs for Brady, AHEPA Foundation, and the Jartrude Fogarty Trust.

Address correspondence to: W. Kimryn Rathmell, 2220 Pierce Avenue, Preston Research Building, Suite 798C, Nashville, Tennessee 37232, USA. Phone: 615.875.9731; Email: Kimryn.Rathmell@VUMC.org.

1. Choueiri TK, et al. Immunomodulatory Activity of Nivolumab in Metastatic Renal Cell Carcinoma. Clin Cancer Res. 2016;22(22):5461-5471.

2. Motzer RJ, et al. Nivolumab plus Ipilimumab versus Sunitinib in Advanced Renal-Cell Carcinoma. NEngl J Med. 2018;378(14):1277-1290.

3. Heng DY, et al. Prognostic factors for overall survival in patients with metastatic renal cell carcinoma treated with vascular endothelial growth factor-targeted agents: results from a large, multicenter study. J Clin Oncol. 2009;27(34):5794-5799.

4. Klapper JA, et al. High-dose interleukin-2 for the treatment of metastatic renal cell carcinoma : a retrospective analysis of response and survival in patients treated in the surgery branch at the National Cancer Institute between 1986 and 2006. Cancer. 2008;113(2):293-301.

5. Motzer RJ, Bacik J, Murphy BA, Russo P, Mazumdar M. Interferon-alfa as a comparative treatment for clinical trials of new therapies against advanced renal cell carcinoma. J Clin Oncol. 2002;20(1):289-296.

6. Varn FS, Wang Y, Mullins DW, Fiering S, Cheng C. Systematic Pan-Cancer Analysis Reveals Immune Cell Interactions in the Tumor Microenvironment. Cancer Res. 2017;77(6):1271-1282.

7. Iglesia MD, Parker JS, Hoadley KA, Serody JS, Perou CM, Vincent BG. Genomic Analysis of Immune Cell Infiltrates Across 11 Tumor Types. J Natl Cancer Inst. 2016;108(11):djw144.

8. Shrimali RK, Yu Z, Theoret MR, Chinnasamy D, Restifo NP, Rosenberg SA. Antiangiogenic agents can increase lymphocyte infiltration into tumor and enhance the effectiveness of adoptive immunotherapy of cancer. Cancer Res. 2010;70(15):6171-6180.

9. Colli LM, Machiela MJ, Myers TA, Jessop L, Yu K, Chanock SJ. Burden of Nonsynonymous Mutations among TCGA Cancers and Candidate Immune Checkpoint Inhibitor Responses. Cancer Res. 2016;76(13):3767-3772.

10. Panda A, et al. Endogenous retrovirus expression is associated with response to immune checkpoint blockade in clear cell renal cell carcinoma. JCI Insight. 2018;3(16):121522. 
11. Smith CC, et al. Endogenous retroviral signatures predict immunotherapy response in clear cell renal cell carcinoma. J Clin Invest. 2018;128(11):4804-4820.

12. Pignon JC, et al. Association of human endogenous retrovirus (hERV) expression with clinical efficacy of PD-1 blockade in metastatic clear cell renal cell carcinoma (mccRCC). J Clin Oncol. 2019;37(suppl_15):4568.

13. Jurka J, Kapitonov VV, Kohany O, Jurka MV. Repetitive sequences in complex genomes: structure and evolution. Annu Rev Genomics Hum Genet. 2007;8:241-259.

14. Lander ES, et al. Initial sequencing and analysis of the human genome. Nature. 2001;409(6822):860-921.

15. Groh S, Schotta G. Silencing of endogenous retroviruses by heterochromatin. Cell Mol Life Sci. 2017;74(11):2055-2065.

16. Schulz WA, Steinhoff C, Florl AR. Methylation of endogenous human retroelements in health and disease. Curr Top Microbiol Immunol. 2006;310:211-250.

17. Chiappinelli KB, et al. Inhibiting DNA Methylation Causes an Interferon Response in Cancer via dsRNA Including Endogenous Retroviruses. Cell. 2015;162(5):974-986.

18. Roulois D, et al. DNA-Demethylating Agents Target Colorectal Cancer Cells by Inducing Viral Mimicry by Endogenous Transcripts. Cell. 2015;162(5):961-973.

19. Brocks D, et al. DNMT and HDAC inhibitors induce cryptic transcription start sites encoded in long terminal repeats. Nat Genet. 2017;49(7):1052-1060.

20. Cañadas I, et al. Tumor innate immunity primed by specific interferon-stimulated endogenous retroviruses. Nat Med. 2018;24(8):1143-1150

21. Laska MJ, et al. Expression of HERV-Fc1, a human endogenous retrovirus, is increased in patients with active multiple sclerosis. J Virol. 2012;86(7):3713-3722.

22. Volkman HE, Stetson DB. The enemy within: endogenous retroelements and autoimmune disease. Nat Immunol. 2014;15(5):415-422.

23. Brodaczewska KK, Szczylik C, Fiedorowicz M, Porta C, Czarnecka AM. Choosing the right cell line for renal cell cancer research. Mol Cancer. 2016;15(1):83.

24. Ricketts CJ, et al. The Cancer Genome Atlas Comprehensive Molecular Characterization of Renal Cell Carcinoma. Cell Rep. 2018;23(12):3698.

25. Cancer Genome Atlas Research Network. Comprehensive molecular characterization of clear cell renal cell carcinoma. Nature. 2013;499(7456):43-49.

26. Solovyov A, et al. Global Cancer Transcriptome Quantifies Repeat Element Polarization between Immunotherapy Responsive and T Cell Suppressive Classes. Cell Rep. 2018;23(2):512-521.

27. Kojima KK. Human transposable elements in Repbase: genomic footprints from fish to humans. Mob DNA. $2018 ; 9: 2$.

28. Rahman M, Jackson LK, Johnson WE, Li DY, Bild AH, Piccolo SR. Alternative preprocessing of RNA-Sequencing data in The Cancer Genome Atlas leads to improved analysis results. Bioinformatics. 2015;31(22):3666-3672.

29. Yoshihara K, et al. Inferring tumour purity and stromal and immune cell admixture from expression data. Nat Commun. 2013;4:2612.

30. Sato Y, et al. Integrated molecular analysis of clear-cell renal cell carcinoma. Nat Genet. 2013;45(8):860-867.

31. Rathmell JC, Farkash EA, Gao W, Thompson CB. IL-7 enhances the survival and maintains the size of naive T cells. J Immunol. 2001;167(12):6869-6876.

32. Siska PJ, et al. Mitochondrial dysregulation and glycolytic insufficiency functionally impair CD8 T cells infiltrating human renal cell carcinoma. JCI Insight. 2017;2(12):93411.

33. Cherkasova E, et al. Detection of an Immunogenic HERV-E Envelope with Selective Expression in Clear Cell Kidney Cancer. Cancer Res. 2016;76(8):2177-2185.

34. Cherkasova E, et al. Inactivation of the von Hippel-Lindau tumor suppressor leads to selective expression of a human endogenous retrovirus in kidney cancer. Oncogene. 2011;30(47):4697-4706.

35. Takeuchi O, Akira S. Pattern recognition receptors and inflammation. Cell. 2010;140(6):805-820

36. Choi MK, et al. A selective contribution of the RIG-I-like receptor pathway to type I interferon responses activated by cytosolic DNA. Proc Natl Acad Sci USA. 2009;106(42):17870-17875.

37. Ranoa DR, et al. Cancer therapies activate RIG-I-like receptor pathway through endogenous non-coding RNAs. Oncotarget. 2016;7(18):26496-26515.

38. Kelley D, Rinn J. Transposable elements reveal a stem cell-specific class of long noncoding RNAs. Genome Biol. 2012;13(11):R107.

39. Kryczek I, et al. Phenotype, distribution, generation, and functional and clinical relevance of Th17 cells in the human tumor environments. Blood. 2009;114(6):1141-1149.

40. Zou W, Wolchok JD, Chen L. PD-L1 (B7-H1) and PD-1 pathway blockade for cancer therapy: Mechanisms, response biomarkers, and combinations. Sci Transl Med. 2016;8(328):328rv4.

41. Racusen LC, et al. Cell lines with extended in vitro growth potential from human renal proximal tubule: characterization, response to inducers, and comparison with established cell lines. J Lab Clin Med. 1997;129(3):318-329.

42. Wang X, Spandidos A, Wang H, Seed B. PrimerBank: a PCR primer database for quantitative gene expression analysis, 2012 update. Nucleic Acids Res. 2012;40(Database issue):D1144-D1149.

43. Dobin A, et al. STAR: ultrafast universal RNA-seq aligner. Bioinformatics. 2013;29(1):15-21.

44. Liao Y, Smyth GK, Shi W. featureCounts: an efficient general purpose program for assigning sequence reads to genomic features. Bioinformatics. 2014;30(7):923-930.

45. Jin Y, Tam OH, Paniagua E, Hammell M. TEtranscripts: a package for including transposable elements in differential expression analysis of RNA-seq datasets. Bioinformatics. 2015;31(22):3593-3599.

46. Love MI, Huber W, Anders S. Moderated estimation of fold change and dispersion for RNA-seq data with DESeq2. Genome Biol. 2014;15(12):550.

47. Subramanian A, et al. Gene set enrichment analysis: a knowledge-based approach for interpreting genome-wide expression profiles. Proc Natl Acad Sci USA. 2005;102(43):15545-15550. 
48. Mootha VK, et al. PGC-1alpha-responsive genes involved in oxidative phosphorylation are coordinately downregulated in human diabetes. Nat Genet. 2003;34(3):267-273.

49. Zhao Y, Ye X, Dunker W, Song Y, Karijolich J. RIG-I like receptor sensing of host RNAs facilitates the cell-intrinsic immune response to KSHV infection. Nat Commun. 2018;9(1):4841.

50. Knuckles P, et al. Drosha regulates neurogenesis by controlling neurogenin 2 expression independent of microRNAs. Nat Neurosci. 2012;15(7):962-969. 